\title{
ON ALGEBRAIC GROUPS AND DISCONTINUOUS GROUPS
}

\author{
TAKASHI ONO
}

To the memory of Tadasi Nakayama

\section{TABLE OF CONTENTS}

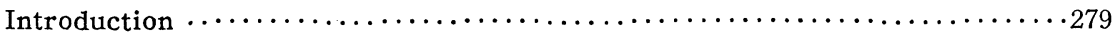

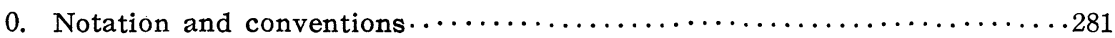

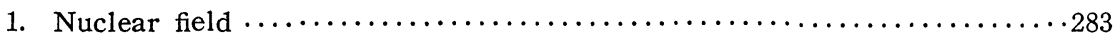

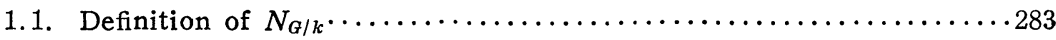

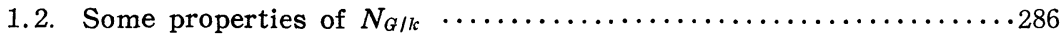

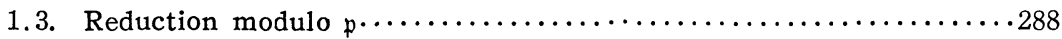

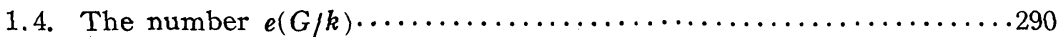

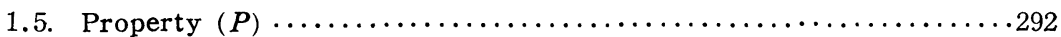

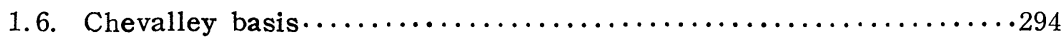

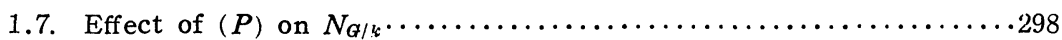

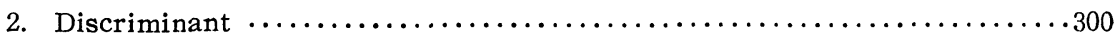

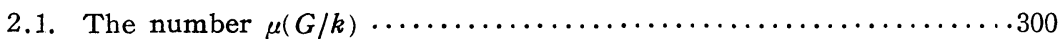

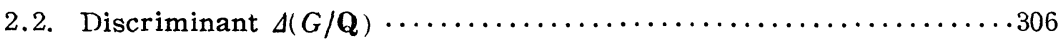

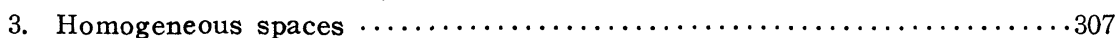

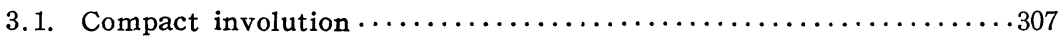

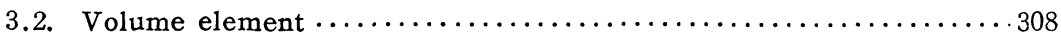

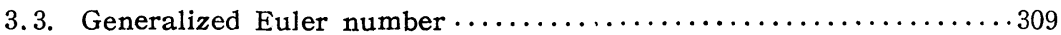

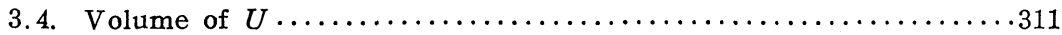

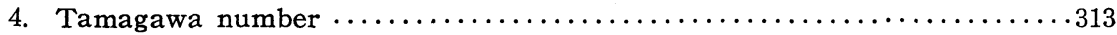

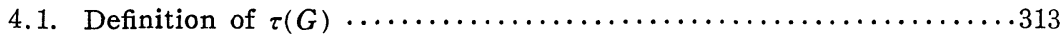

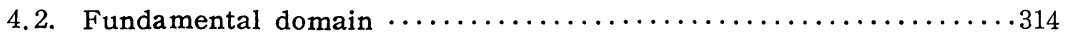

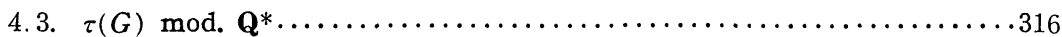

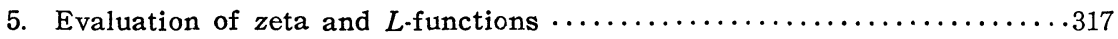

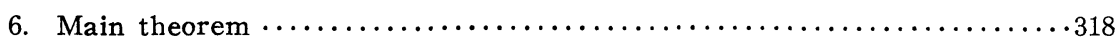

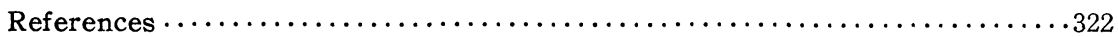

\section{Introduction}

Let $G$ be a connected semi-simple algebraic group defined over $\mathbf{Q}$ and let $\Gamma$ be a discrete subgroup of $G_{\mathrm{R}}$ (the subgroup of $G$ consisting of points rational over $\mathbf{R})$ such that $\Gamma \backslash G_{\mathbf{R}}$ is compact. The main purpose of the present paper is to prove that for a certain type of group $G$ there exists an invariant algebraic

Received June 15, 1965. 
differential from $\omega$ on $G$ of highest degree defined over $\mathbf{Q}$ such that

$$
\int_{\Gamma \backslash G_{\mathbf{R}}} \omega_{\mathbf{R}}=\Pi_{\nu=1}^{l} L_{\nu}\left(a_{\nu}\right)
$$

where $a_{\nu}, 1 \leqq \nu \leqq l$, are integers such that the Poincare polynomial of the compact from of $G$ is $\Pi_{\nu=1}^{l}\left(t^{2 a_{\nu}-1}+1\right), L_{\nu}, 1 \leqq \nu \leqq l$, are $L$-functions determined by $G$ and $\omega_{\mathbf{R}}$ is the analytic differential form on $G_{\mathbf{R}}$ induced by $\omega$.

If, in particular, one takes an arithmetic group as $\Gamma$, the relation $(*)$ is equivalent to the rationality of the Tamagawa number $\tau(G)$ for a certain type of group $G$. This is Theorem 6.6.

Let us now explain what is meant by a certain type of $G$. Let $G_{0}$ be the topological identity component of $G_{\mathbf{R}}$, let $X$ be the associated symmetric space: $X=G_{0} / K, K$ being a maximal compact subgroup of $G_{0}$, and let $X_{\mathfrak{u}}$ be the compact form of $X$.

First of all, we impose on $G$ the condition that the gaussian curvature of $X$ is different from zero, or equivalently, that the Euler number of $X_{u}$ is strictly positive. We shall call this condition $(P)$. By $(P)$, the computation of the integral in $(*)$ is reduced to the Gauss-Bonnet theorem (cf. (3.3.10)). Incidentally, when $X$ admits the hermitian structure, $(P)$ is automatically satisfied.

Second of all, we have to exclude the worst twisted group, i.e. the trialitarian form of the second kind of type $D_{4}$; for this case the relation (*) should be modified (as for the details, see $\S 6$ ). These are restrictions on $G$.

On the other hand, as many people believe, one can probably drop the compactness assumption of $\Gamma \backslash G_{\mathbf{R}}$ by showing that the value of the curvature integral ( = the generalized Euler number) is rational whenever $\Gamma \backslash G_{\mathbf{R}}$ is of finite volume, as Satake has done for symplectic groups [21]; but we shall not go further in this direction in this paper.

As for the $L$-fuctions in $(*)$, we have to explain what Galois extension they belong to. Without loss of generality, we may assume that $G$ is $\mathbf{Q}$-simple, i.e. $G$ has no proper normal subgroup defined over $\mathbf{Q}$. Then, if we call $k$ the smallest field of definition for an absolutely simple factor $\widetilde{G}$ of $G$, we see at once that $G$ is isogenous over $\mathbf{Q}$ to the group $R_{k / Q}(\widetilde{G})$ obtained from $\widetilde{G}$ by restricting the field of definition from $k$ to $\mathbf{Q}$. For $\widetilde{G}$, we can attach a finite Galois extension $N_{\tilde{G} / k}$ (called nuclear field $(\S 1)$ ) whose Galois group is imbedded in the group of automorphims of the Dynkin diagram of $\widetilde{G}$. The $L$ - 
functions are then taken from the Galois extension $N_{\tilde{G} / k}$ over $k$ (1.3).

Now, the condition $(P)$ imposed on $G$ takes two parts in this work. On the one hand, under $(P)$, one can use the Gauss-Bonnet theorem for $\Gamma \backslash X$ and $X_{u}$, effectively; on the other hand, $(P)$ implies that the fields $k, N_{\tilde{G} / k}$ above become totally real or totally imaginary; and this fact, together with Weil's result : $\tau(S U(n))=1$, makes possible the evaluation (mod. $\mathbf{Q}^{*}$ ) of the $L$-functions at $\left.a_{\nu}, 1 \leqq \nu \leqq l,(\S 5)^{*}\right)$.

After these preparations, it is not hard to see that the trancendental factors in both hand sides of $(*)$ are powers of $\pi$ with the same exponent. In order to finish the proof of $(*)$ one has to take care of a quadratic number $\Delta(G / \mathbf{Q})^{1 / 2}$ $(\S 2)$, which seems to us interesting by itself.

As the readers notice, the quantities in the both hand sides of $(*)$ are irrelevant to the symmetric space $X$ and hence to the condition $(P)$. It is quite desirable to prove the equality of type $(*)$ without any assumption on $G$. A typical example is the case $G=S L(n), n \geqq 3$, where $G$ is not of type $(P)$ and $G_{\mathbf{Z}} \backslash G_{\mathbf{R}}$ is not compact, and it is well known that the integral (with respect to a suitable $\omega$ ) is precisely equal to the product of (Riemann) zeta functions evaluated at $2,3, \ldots, n$ (Minkowski Siegel).

Finally, it is interesting that an invariant of $G$ such as the volume of the fundamental domain decomposes in the same manner as the corresponding compact form decomposes into the product of spheres homologically. This would raise a question: can one define a zeta function $\zeta_{G}$ for $G$ so that the way of decomposition of $\zeta_{G}$ into ordinary $L$-functions is the same as the way of decomposition of the compact form into spheres?

\section{Notation and conventions}

0.1. As usual, $\mathbf{Z}, \mathbf{Q}, \mathbf{R}, \mathbf{C}$ are integers, rational numbers, real numbers and complex numbers, respectively. We shall further use the following notation:

* At this stage I owe a great deal to Shimura; he persuaded me to check every detail of my method for the case of special unitary group $S U(\boldsymbol{n})$ relative to the quadratic extension $K / F$, where $K$ is totally imaginary and $F$ is totally real. After having done this experiment, I could find the second meaning of $(P)$ mentioned above. Incidentally, it turned out that among all groups for which $\tau(G)$ is known, $S U(n)$ is the richest group by which one can evaluate zeta or $L$-functions in the sense of $\$ 5$, It seems impossible to find out e.g. $\zeta_{\mathbf{Q}}(3)$ by using algebraic groups, 
$\mathbf{F}_{q}$ : the finite field with $q$ elements

$\mathbf{Q}_{p}$ : the $p$-adic number field for a prime number $p$

$\mathbf{Z}_{p}$ : the ring of $p$-adic integers

$G_{a}$ : the additive group of the universal domain

$G_{m}$ : the multiplicative group of the universal domain

$\bar{K}$ : the algebraic closure of a field $K$

$K^{*}$ : the multiplicative group of a field $K$

$g(K / k)$ : the Galois group of the Galois extension $K / k$

Aut $X$ : the group of automorphisms of a structure $X$

Inn $G$ : the group of inner automorphisms of a group $G$

[S] : the cardinality of a set $S$

0.2. In the group $\mathbf{C}^{*}$, when two numbers $a, b$ are congruent modulo the subgroup $\mathbf{Q}^{*}$, we shall write $a \sim b$.

0.3. Let $\Omega$ be a universal domain, $M(n)$ be the associative algebra of matrices of degree $n$ over $\Omega$. For any subring $\Lambda$ (with 1 ) of $\Omega, M(n)_{\Lambda}$ will denote the subring of $M(n)$ consisting of matrices with coefficients in $\Lambda$. The group of units of $M(n)_{\Lambda}$ will be denoted by $G L(n)_{\Lambda}$. In particular, we put $G L(n)=G L(n)_{\Omega}$. When we view $M(n)_{\Lambda}$ as Lie algebra over $\Lambda$ by $[X, Y]=$ $X Y-Y X$, we denote it by $\mathfrak{g l}(n)_{\Lambda}$ and put $\mathfrak{g l}(n)=\mathfrak{g l}(n)_{\Omega}$. Given a subgroup $G$ of $G L(n)$, we put $G_{\Lambda}=G \cap G L(n)_{\Lambda}$. The same convention will be applied to a subalgebra $g$ of $g \mathfrak{l}(n)$, i.e. $g_{\Lambda}=g \cap g \mathfrak{l}(n)_{\Lambda}$.

0.4. Let $\Omega$ be of characteristic zero. To each $X \in \mathfrak{g l}(\boldsymbol{n})$ there corresponds a derivation $\delta(X)$ of the polynomial ring $\Omega[\xi], \xi=\left(\xi_{i j}\right)_{1 \leqq i, j \leqq n}$ given by

$$
\left.\delta(X) P=\operatorname{tr}^{t}(\operatorname{grad} \xi P)(\xi X)\right),
$$

where $\operatorname{grad}_{a} P$ means the matrix $\left(\frac{\partial P}{\partial \xi_{i j}}\right)$ evaluated at $\xi=a$. Let $G$ be a connected algebraic group in $G L(n)$ defined over a field $k$, let $I(G)$ be the prime ideal in $\Omega[\xi]$ determined by $G$. Since the map $X \rightarrow \delta(X)$ is a homomorphism as Lie algebras,

$$
\mathfrak{g}=\{X \in \mathfrak{g l}(n), \delta(X) I(G) \subseteq I(G)\}
$$

becomes a Lie algebra, i.e. the matric Lie algebra of $G$ in the sense of Cheyalley [8, Chap. II $\S 8]$. Since $G$ is defined over $k, g$ is defined over $k$, or, equivalently, $g$ admits a basis in $g l(n)_{k}$. Let $\Omega(G)$ be the function field for $G$, i.e. the field 
of quotients of $\Omega[\xi] / I(G)$. From (0.4.1), $\delta(X)$ induces a derivation $\delta_{\mathfrak{g}}(X)$ of $\Omega(G)$. Denote by $D$ the Lie algebra over $\Omega$ of all left invariant derivations of $\Omega(G)$. Then $\delta_{\mathfrak{g}}$ gives an isomorphism $\delta_{\mathfrak{g}}: \mathfrak{g} \simeq \mathscr{D}$ and hence induces an isomorphism

$$
\left(\delta_{\mathfrak{g}}\right)_{k}: \mathfrak{g}_{k} \widetilde{\mathfrak{D}_{k}},
$$

where $\mathfrak{D}_{k}$ means the set of derivations defined over $k$.

Assuming $k=\mathbf{R}$, denote by $G_{0}$ the topological identity component of the real Lie group $G_{\mathrm{R}}$, and by $g_{0}$ the Lie algebra of $G_{0}$ in the analytic sense. There is an isomorphism over $\mathbf{R}$

$$
\alpha: g_{0} \underset{\mathfrak{g}}{\longrightarrow} \mathfrak{g}_{\mathbf{R}}
$$

given by $\alpha(X)=\left(X_{e} x_{i j}\right)_{1 \leqq i, j \leqq n}$, where $X$ is a left invariant vector field on $G_{0}$ and $x_{i j}$ are the coordinate functions on $G_{0}$. Through (0.4.2), (0.4.3), we shall often identify isomorphic Lie algebras. E.g. let $\left\{X_{1}, \ldots, X_{d}\right\}$ be a basis of $g_{Q}$, where $g$ is the matric Lie algebra of an algebraic group defined over $\mathbf{Q}$. Viewing $X_{i}^{\prime} s$ as a basis for $\mathfrak{D}_{Q}$, the dual basis $\omega_{i}, 1 \leqq i \leqq d$, is a basis for left invariant algebraic 1 -forms on $G$ defined over $\mathbf{Q}$, and $\omega=\omega_{1} \wedge \cdots \wedge \omega_{d}$, is a left invariant algebraic $d$-form on $G$, defined over $\mathbf{Q}$. On the other hand, viewing $X_{i}^{\prime} s$ as a basis for $g_{0}$, the same procedure gives a left invariant analytic $d$-form $\omega_{\mathbf{R}}$ on $G_{0}$. We shall again denote by $\omega_{\mathbf{R}}$ the Haar measure on $G_{0}$ determined by it. In the text, we shall call either $\omega$ or $\omega_{\mathbf{R}}$ the $d$-form which is the product of 1 -forms dual to $X_{i}, 1 \leqq i \leqq d$.

\section{Nuclear field}

\section{1. Definition of $\boldsymbol{N}_{G / k}$}

We first recall the non-infinitesimal approach given in [10]. Let $G$ be a connected semi-simple algebraic group, $T$ be a maximal torus of $G$ and $B$ be a Borel subgroup of $G$ containing $T$. Denote by $\hat{T}$ the character module Hom $\left(T, G_{m}\right)$. A character $\alpha \in \hat{T}$ will be called a root of $G$ with respect to $T$ if there exists an isomorphism $x_{x}$ of $G_{a}$ with a subgroup $\mathscr{L}_{\alpha}$ of $G$ such that

$$
t x_{\alpha}(\lambda) t^{-1}=x_{\alpha}(\alpha(t) \lambda), \quad t \in T, \lambda \in G_{a} .
$$

This condition implies that $\alpha \neq 0$ and that $x_{\alpha}$ is uniquely determined up to a non-zero scalar multiplication in $G_{a}$. We denote by $\Delta$ the set of all roots of 
$G$ with respect to $T$. We define a subset $\Delta_{B}$ of $\Delta$ by

$$
\Delta_{B}=\left\{\alpha \in \Delta, \mathscr{Y}_{\alpha} \subset B\right\} .
$$

Since $\Delta_{B}$ satisfies the condition for positive roots, we can define a subset $\Pi$ of $\Delta_{B}$ consisting of fundamental roots and further the Dynkin diagram $D$, with respect to $T, B$.

Let $f$ be an isomorphism of another algebraic group $G^{\prime}$ with $G$ and put $T^{\prime}=f^{-1}(T), B^{\prime}=f^{-1}(B)$. Let $\Delta^{\prime}, \Delta^{\prime} B^{\prime}, \Pi^{\prime}$ be to $G^{\prime}$ with respect to $T^{\prime}, B^{\prime}$, what $\Delta, \Delta_{B}, \Pi$ were to $G$. Denote by $\hat{f}$ the isomorphism $\hat{T} \rightarrow \hat{T}^{\prime}$ given by

$$
\hat{f}(\xi)\left(t^{\prime}\right)=\xi\left(f\left(t^{\prime}\right)\right), \quad t^{\prime} \in T^{\prime}, \xi \in \hat{T} .
$$

One verifies easily that the map $x_{\hat{f}(\alpha)}=f^{-1} x_{\alpha}$ satisfies the condition (1.1.1) for $\hat{f}(\alpha)$ and that $\hat{f}(\Delta)=\Delta^{\prime}, \hat{f}\left(\Delta_{B}\right)=\Delta^{\prime}{ }^{\prime}, \hat{f}(\Pi)=\Pi^{\prime}$. If, in particular, $f$ is an automorphism of $G$ such that $f(T)=T$ and $f(B)=B$, then $f$ induces an automorphism of the Dynkin diagram $D$. We shall denote by $f_{*}$ the inverse of this induced automorphism. For a general $f \in$ Aut $G$, one can find an inner automorphism $u$ of $G$ such that $u f(T)=T, \imath f(B)=B$, Then $(u f)_{*} \in$ Aut $D$ is independent of the choice of $u[10,17-07$, Prop. 1]. Hence, we can define $f_{*} \in$ Aut $D$ for any $f \in$ Aut $G$ by $f_{*}=(u f)_{*}$. One then sees that the map $f \rightarrow f_{*}$ is a homomorphism of Aut $G$ into Aut $D$ whose kernel is Inn $G$.

Let $k$ be a perfect field. We say that a connected semi-simple algebraic group $G$ defined over $k$ is a group of Chevalley type over $k$ if $G$ admits a maximal torus which is trivial over $k$ (or $k$-trivial, for short). It is known that if $G$ is of Chevalley type over $k$ then every Borel subgroup containing a $k$-trivial maximal torus is defined over $k$ [17].

Proposition 1.1.2. Let both of $G, G^{\prime}$ be groups of Chevalley type over a perfect field $k$. Let $\psi$ be an isomorphism defined over $\bar{k}$ of $G^{\prime}$ with $G$. Then $\psi^{\wedge} \psi^{-1}$ is an inner automorphism of $G$ for every $\sigma \in \mathfrak{g}(\bar{k} / k)$.

In fact, let $T, T^{\prime}$ be $k$-trivial maximal tori of $G, G^{\prime}$, respectively. There is then an inner automorphism $u$, defined over $\bar{k}$, of $G$ such that $u \psi\left(T^{\prime}\right)=T$. Put $\phi=u \psi$ and $B=\phi\left(B^{\prime}\right)$, where $B^{\prime}$ is any Borel subgroup of $G^{\prime}$ containing $T^{\prime}$. By the above remark, all groups $T, T^{\prime}, B, B^{\prime}$ are defined over $k$. Hence, the automorphism $\phi^{\sigma} \phi^{-1}$ of $G$ conserves $T, B$ for any $\sigma \in \mathfrak{g}(\bar{k} / k)$. However, by the assumption on $T, T^{\prime}$, the action of $g(\bar{k} / k)$ on $\hat{T}, \hat{T}^{\prime}$ is trivial, and so $\hat{\phi}^{\sigma}=(\hat{\phi})^{\sigma}$ 
$=\hat{\phi}$, from this follows that $\phi^{\sigma} \phi^{-1} \in \operatorname{Inn} G$, and hence $\phi^{\sigma} \phi^{-1}=u^{-\sigma} \phi^{\top} \phi^{-1} u \in \operatorname{Inn} G$, q.e.d.

CoRollaki 1.1.3. Let $G$ be a group of Chevalley type over $k$. For any $f \in \operatorname{Aut}_{\bar{k}} G$ (automorphisms defined over $\bar{k}$ ), $f^{\sigma} f^{-1}$ is inner for any $\sigma \in g(\bar{k} / k)$. In other words, we have $\left(f^{\sigma}\right)_{*}=f_{*}, \sigma \in g(k / k)$, where $*$ is defined relative to a $k$-trivial maximal torus and a Borel subgroup containing it.

As is well-known, given a connected semi-simple algebraic group $G$ defined over a perfect field $k$, there is an isomorphism $\phi$ defined over $\vec{k}$ of $G$ with a group $\mathbf{G}$ of Chevalley type over $k$.

$$
\phi: G \widetilde{G} .
$$

Put $f_{\sigma}=\phi^{\wedge} \phi^{-1} \in$ Aut G. Then one has

$$
f_{\sigma \tau}=f_{\sigma}^{\tau} f_{\tau}, \quad \sigma, \tau \in g(\bar{k} / k) .
$$

From (1.1.3) we see that the map $\sigma \rightarrow\left(f_{\sigma}\right)_{*}$ is a homomorphism of $g(\bar{k} / k)$ into Aut D. We claim that the kernel of this homomorphism is independent of the choice of $\mathbf{G}$ and $\phi$. In fact, let $\phi^{\prime}: G \widetilde{G} \rightarrow \mathbf{G}^{\prime}$ be another isomorphism over $\bar{k}$ of $G$ with a group $\mathbf{G}^{\prime}$ of Chevalley type over $k$ and put $f_{\sigma}^{\prime}=\phi^{\prime \sigma} \phi^{\prime-1}$ and $\psi=\phi \phi^{\prime-1}$. By (1.1.2) one has $\left(\phi^{\sigma} \phi^{-1}\right)_{*}=$ id., and so $\left(f_{\sigma}\right)_{*}=\left(\phi^{\sigma} \phi^{-1}\right)_{*}=\left(\left(\phi \phi^{\prime}\right)^{\sigma}\left(\phi \phi^{\prime}\right)^{-1}\right)_{*}=$ $\left(\phi^{\sigma} \psi^{-1}\right)_{*}\left(\phi f_{\sigma}^{\prime} \psi^{-1}\right)_{*}=\left(\phi f_{\sigma}^{\prime} \psi^{-1}\right)_{*}$, from this follows our assertion. Call $\mathfrak{n}_{G / k}$ the kernel of $\sigma \rightarrow\left(f_{\sigma}\right)_{*}$. As Aut $D$ is a finite group, $\mathfrak{n}_{G / k}$ has a finite index in $g(\bar{k} / k)$. We shall denote by $N_{G / k}$ the corresponding finite Galois extension over $k$ and call it the nuclear field for $G$ over $k$. Thus the Galois group of $N_{G / k}$ over $k$ is imbedded in Aut $D$. The next proposition shows that $N_{G / k}$ is not only invariantly attached to $G$ over $k$ but is unchanged under the isogeny.

Proposition 1.1.4. Let $k$ be a perfect field and let $f: G^{\prime} \rightarrow G$ be an isogeny defined over $k$ of connected semi-simple algebraic groups $G, G^{\prime}$ defined over $k$. Then one has $N_{G / k}=N_{\theta^{\prime} / k}$.

In fact, let $\phi^{\prime}$ be an isomorphism defined over $\bar{k}$ of $G^{\prime}$ with a group $\mathbf{G}^{\prime}$ of Chevalley type over $k$. Since $M=\phi^{\prime}(\operatorname{Ker} f)$ is central in $\mathbf{G}^{\prime}, M$ is contained in any one of $k$-trivial maximal torus of $\mathbf{G}^{\prime}$, and so $M$ is imbedded in a product of a certain number of the group of roots of unity of a finite Galois extension of $k$. From this we see that $M$ is defined over $k$ as a zero dimensinal algebraic group. So we get an isogeny over $k: g: \mathbf{G}^{\prime} \rightarrow \mathbf{G}=\mathbf{G}^{\prime} / M$, this factor group being 
of Chevalley type over $k$, and an isomorphism $\phi: G \Im \mathbf{G}$ such that $\phi f=g \phi^{\prime}$. Now, put $f_{\sigma}=\phi^{\circ} \phi^{-1}, f_{\sigma}^{\prime}=\phi^{\prime \sigma} \phi^{\prime-1}$. Then, $f_{\sigma}$ lies under $f_{\sigma}^{\prime}$ with respect to the covering $\left(\mathbf{G}^{\prime}, g\right)$ over $\mathbf{G}$. One has to verify that

$$
f_{\sigma} \in \operatorname{Inn} \mathbf{G} \Longleftrightarrow f_{\sigma}^{\prime} \in \operatorname{Inn} \mathbf{G}^{\prime} \text {. }
$$

The implication $(\Leftarrow)$ is trivial. Suppose next that $f_{\sigma}(x)=a x a^{-1}, a \in \mathbf{G}$. Take an $a^{\prime} \in \mathbf{G}^{\prime}$ such that $g\left(a^{\prime}\right)=a$. Then, one has $f_{\sigma}^{\prime}\left(x^{\prime}\right)=\zeta\left(x^{\prime}\right) a^{\prime} x^{\prime} a^{\prime-1}, x^{\prime} \in \mathbf{G}^{\prime}$, where $\zeta\left(x^{\prime}\right)$ are contained in the center of $\mathbf{G}^{\prime}$. However, $\zeta$ must be trivial because it is a homomorphism of the connected group into a finite group, which proves $(\Longrightarrow)$.

PROPOSITION 1.1.5. Let $G$ be a connected semi-simple algebraic group defined over a perfect field $k$ and let $E$ be a perfect field containing $k$. Then, one has $N_{G / E}=E N_{G / k}$, the compositum of $E$ and $N_{G / k}$.

In fact, let $\phi$ be an isomorphism defined over $\bar{k}$ of $G$ with a group $\mathbf{G}$ of Chevalley type over $k$. For a $\sigma \in \mathfrak{g}(\bar{E} / E)$, we have:

$\sigma$ leaves $N_{G / E}$ elementwise fixed $\Longleftrightarrow \sigma \in \mathfrak{n}_{G / E}$

$\Longleftrightarrow \phi^{\sigma} \phi^{-1} \in \operatorname{Inn} \mathbf{G} \Longleftrightarrow \sigma \mid \bar{k}$ ( $\sigma$ restricted on $\left.\bar{k}\right) \in \mathfrak{n}_{G / k}$

$\Leftrightarrow \sigma \mid \vec{k}$ leaves $N_{G / k}$ elementwise fixed

$\Leftrightarrow \sigma$ leaves $E N_{G / k}$ elementwise fixed, which proves our assertion.

Proposition 1.1.6. Let $k$ be a perfect field and $K$ be a finite extension of k. Let $H$ be a connected semi-simple algebraic group defined over $K$ and let $G=R_{K / k}(H)$, the algebraic group defined over $k$ obtained from $H$ by restricting the field of definition $K$ to $k$ (cf. [25, p. 4]). Let $N_{G / k}\left(\right.$ resp. $\left.N_{H / K}\right)$ be the nuclear field for $G$ (resp. $H$ ) over $k$ (resp. K). Then, $N_{G / k}$ contains $N_{H / K}$, and $N_{G / k}$ is the smallest Galois extension of $k$ containing $N_{H / K}$.

The proof will be left to the reader.

\subsection{Some properties of $N_{G / k}$}

Let $G$ be a connected semi-simple algebraic group defined over a perfect field $k$. By a splitting field for $G$ over $k$ we shall mean an algebraic extension $K$ of $k$ over which $G$ is isomorphic with a group of Chevalley type over $k$.

Proposition 1.2.1. The nuclear field $N_{G / k}$ is contained in any splitting field for $G$ over $k$. 
In fact, let $\mathfrak{h}$ be a subgroup of $g(\bar{k} / k)$ corresponding to a splitting field $K$ for $G$ over $k$ and let $\phi: G \simeq \mathbf{G}$ be an isomorphism defined over $K$ of $G$ with a group G. of Chevalley type over $k$. Then, for $\sigma \in \mathfrak{h}$, we have $f_{\sigma}=\phi^{\top} \phi^{-1}=\mathrm{id}$., and so $\sigma \in \mathfrak{n}_{\alpha / k}$, q.e.d.

The following proposition will give a partial converse of (1.2.1).

PROPOSITION 1.2.2. If $k$ is finite, the nuclear field $N_{G / k}$ is a splitting field for $G$ over $k$.

In fact, let $\phi$ be an isomorphism defined over $\bar{k}$ of $G$ with a group $\mathbf{G}$ of Chevalley type over $k$. Let $Z$ be the center of $\mathbf{G}$. Hence $\operatorname{Inn}_{k} \mathbf{G}$ (inner automorphisms defined oner $\bar{k})$ is identified with $(\mathbf{G} / Z)_{\bar{k}}$. If we restrict the cocycle $f_{\sigma}=\phi^{\sigma} \phi^{-1}$ on $\mathfrak{n}_{G / k}$, it becomes a cocycle of $g\left(\bar{k} / N_{G / k}\right)$ in $(\mathbf{G} / Z)_{\bar{k}}$ which is trivial by Lang's theorem [13] applied to the connected algebraic group $\mathbf{G} / Z$. So, there is a $u \in \operatorname{Inn}_{\bar{k}} G$ such that $f_{\sigma}=u^{\sigma} u^{-1}, \sigma \in \mathfrak{g}\left(\bar{k} / N_{a: k}\right)$ and hence $u^{-1} \phi$ is defined over $N_{G / k}$, i.e. $N_{G / k}$ is a splitting field for $G$ over $k$.

Remark 1.2.3. When $k$ is finite, the above two propositions show that $N_{G / k}$ is the smallest splitting field for $G$ over $k$. If $G$ is simple, $N_{G / k}$ is at most quadratic or cubic extension of $k$. The quadratic extension can happen only for groups of type $A_{l}(l \geqq 2) D_{l}(l \geqq 4)$ and $E_{6}$. The cubic extension can happen only for $D_{4}$. Let $q$ be the number of elements in $k: k=\mathbf{F}_{q}$. The-following table which gives the order of $G_{\mathrm{F}_{q}}$ is due to Chevalley and Steinberg $[9,24]$

TABLE 1

\begin{tabular}{|c|c|c|}
\hline type & {$\left[N_{G / \mathbf{F}_{q}:}: \mathbf{F}_{q}\right]$} & {$\left[G_{\mathrm{F}_{q}}\right]$} \\
\hline$A_{l}(l \geqq 1)$ & 1 & $q^{l(l+1) / 2} \Pi_{\nu=1}^{l}\left(q^{\nu+1}-1\right)$ \\
\hline$A_{l}(l \geqq 2)$ & 2 & $q^{l(l+1) / 2} \Pi_{\nu=1}^{l}\left(q^{\nu+1}-(-1)^{\nu+1}\right)$ \\
\hline$B_{l}(l \geqq 2)$ & 1 & $q^{l^{2} \Pi_{\nu=1}^{l}\left(q^{2 \nu}-1\right)}$ \\
\hline$C_{l}(l \geqq 3)$ & 1 & $q^{l^{2}} \Pi_{\nu=1}^{l}\left(q^{2 \nu}-1\right)$ \\
\hline$D_{l}(l \geqq 4)$ & 1 & $q^{l(l-1)}\left(q^{l}-1\right) \Pi_{\nu=1}^{l-1}\left(q^{2 \nu}-1\right)$ \\
\hline$D_{l}(l \geqq 4)$ & 2 & $q^{l l l-1)}\left(q^{l}+1\right) \Pi_{\nu=1}^{l-1}\left(q^{2 \nu}-1\right)$ \\
\hline$D_{4}$ & 3 & $q^{12}\left(q^{4}-\omega\right)\left(q^{2}-1\right)\left(q^{4}-\bar{\omega}\right)\left(q^{6}-1\right)$ \\
\hline$E_{6}$ & 1 & $q^{36}\left(q^{2}-1\right)\left(q^{5}-1\right)\left(q^{6}-1\right)\left(q^{8}-1\right)\left(q^{9}-1\right)\left(q^{12}-1\right)$ \\
\hline$E_{6}$ & 2 & $q^{36}\left(q^{2}-1\right)\left(q^{5}+1\right)\left(q^{6}-1\right)\left(q^{3}-1\right)\left(q^{9}+1\right)\left(q^{12}-1\right)$ \\
\hline$E_{7}$ & 1 & $q^{63}\left(q^{2}-1\right)\left(q^{6}-1\right)\left(q^{8}-1\right)\left(q^{10}-1\right)\left(q^{12}-1\right)\left(q^{14}-1\right)\left(q^{18}-1\right)$ \\
\hline$E_{8}$ & 1 & $q^{120}\left(q^{2}-1\right)\left(q^{8}-1\right)\left(q^{12}-1\right)\left(q^{14}-1\right)\left(q^{18}-1\right)\left(q^{20}-1\right)\left(q^{24}-1\right)\left(q^{30}-1\right)$ \\
\hline$F_{4}$ & 1 & $q^{24}\left(q^{2}-1\right)\left(q^{6}-1\right)\left(q^{8}-1\right)\left(q^{12}-1\right)$ \\
\hline$G_{2}$ & 1 & $q^{6}\left(q^{2}-1\right)\left(q^{6}-1\right)$ \\
\hline
\end{tabular}


and will play an important role in this paper.

When $\left[N_{G / \mathbf{F}_{q}}: \mathbf{F}_{q}\right]=1$, i.e. when $G$ is already of Chevalley type over $\mathbf{F}_{q}$, the expression of $\left[G_{\mathbf{F}_{q}}\right]$ in the above table is unified in the following formula which is valid for any connected semi-simple group $\mathbf{G}$ of Chevalley type over $\mathbf{F}_{q}:$

$$
\left[\mathbf{G}_{\mathbf{F}_{q}}\right]=q^{N} \Pi_{\nu=1}^{l}\left(q^{a \nu}-1\right),
$$

where $N$ is the number of positive roots, $l$ is the rank ( = the dimension of the maximal torus) and $a_{\nu}$ are integers such that the Poincare polynomial of the compact form of the complex semi-simple Lie algebra belonging to the same type as $\mathbf{G}$ is $\Pi_{\nu=1}^{l}\left(t^{2 a_{\nu}-1}+1\right)$ (cf. [9]).

\subsection{Reduction modulo $p$}

Let $k$ be an algebraic number field of finite degree over $\mathbf{Q}$ (a number field, for short), $G$ be a connected semi-simple algebraic group defined over $k$ and let $N_{G / k}$ be the nuclear field for $G$ over $k$. We denote by $G^{(\mathfrak{p})}$ the algebraic group defined over the residue field $k^{(\mathfrak{p})}$ at a prime ideal $\mathfrak{p}$ in $k$ obtained from $G$ by the reduction modulo $\mathfrak{p}$. For almost all $\mathfrak{p}, G^{(\mathfrak{p})}$ remains connected semi-

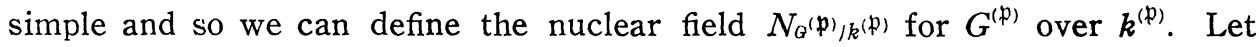
$K$ be a finite Galois splitting field for $G$ over $k$ and $\phi$ be an isomorphism, defined over $K$, of $G$ with a group $\mathbf{G}$ of Chevalley type over $k$. By (1.2.1), $N_{a / k}$ is contained in $K$. Take a $k$-trivial maximal torus $T$ of $\mathbf{G}$ and a Borel subgroup $B$ containing $T$. Denote by $D$ the Dynkin diagram of $\mathbf{G}$ with respect to $T, B$. For almost all $\mathfrak{p}, T^{(\mathfrak{p})}, B^{(\mathfrak{p})}$ remain $k^{(\mathfrak{p})}$-trivial maximal torus and a Borel subgroup of the group $\mathbf{G}^{(\mathfrak{p})}$ of Chevalley type over $k^{(\mathfrak{p})}$. Denote by $D^{(\mathfrak{p})}$ the Dynkin diagram of $G^{(\mathfrak{p})}$ with respect to $T^{(\mathfrak{p})}, B^{(\mathfrak{p})}$. Since every character in $\hat{T}$ is defined over $k$, one can define the reduction modulo $p$ of any finite number of characters, for almost all $\mathfrak{p}$. In particular, one sees that, for almost all $\mathfrak{p}$, the map $\alpha \rightarrow \alpha^{(\mathfrak{p})}$ induces a one-to-one correspondence of the systems of fundamental roots for $\mathbf{G}$ and $\mathbf{G}^{(\mathfrak{p})}$, and thus gives rise to an isomorphism of $D$ and $D^{(\mathfrak{)})}$ as Dynkin diagrams. Let $\mathbf{P}$ be a prime ideal of $K$ lying above $g$. For almost all $\mathfrak{p}$, the reduced map $\phi^{(\mathbf{P})}$ which is defined over the residue field $K^{(\mathbf{P})}$ gives an isomorphism of $G^{(\mathfrak{p})}$ with $\mathbf{G}^{(\mathfrak{p})}$. For an $f \in \mathrm{Aut}_{K} \mathbf{G}$, one has

$$
\left(f_{*}(\alpha)\right)^{(\mathfrak{p})}=\left(f^{(\mathbf{P})}\right)_{*}\left(\alpha^{(\mathfrak{p})}\right), \quad \alpha \in D,
$$

for almost all $\mathfrak{p}$. In the following argument, we shall often omit the phrase 
"for almost all p" and so all sentences will make sense only for almost all primes which do not disturb the smooth argument. Denote by $Z_{\mathrm{P}}$ the decomposition field of $\mathbf{P}$ relative to $K / k$. There is a canonical isomorphism

$$
\rho: g\left(K / Z_{\mathbf{P}}\right) \sim g\left(K^{(\mathbf{P})} / k^{(\mathfrak{p})}\right)
$$

by which corresponds the Frobenius automorphisms. Put $f_{\sigma}=\phi^{\top} \phi^{-1}, \sigma \in g(K / k)$ and $F_{s}=\left(\phi^{(\mathbf{P})}\right)^{s}\left(\phi^{(\mathbf{P})}\right)^{-1}, s \in g\left(K^{(\mathbf{P})} / k^{(\mathbf{P})}\right)$. In view of the formula

$$
\left(\phi^{\jmath}\right)^{(\mathbf{P})}=\left(\phi^{(\mathbf{P})}\right)^{p(\sigma)}, \quad \sigma \in \mathcal{g}\left(K / Z_{\mathbf{P}}\right),
$$

we get

$$
F_{\rho(\sigma)}=\left(f_{\sigma}\right)^{(\mathbf{P})}, \quad \sigma \in \mathfrak{g}\left(K / Z_{\mathbf{P}}\right) .
$$

Call $\xi$ the map $g(K / k) \rightarrow$ Aut $D$ given by $\xi(\sigma)=\left(f_{\sigma}\right)_{*}$ and denote by $\xi_{\mathbf{P}}$ the restriction of $\xi$ on the decomposition group $g\left(K / Z_{\mathrm{P}}\right)$. On the other hand, call $\eta$ the map $g\left(K^{(\mathbf{P})} / k^{(\mathfrak{p})}\right) \rightarrow$ Aut $D^{(\mathfrak{p})}$ given by $\eta(s)=\left(F_{s}\right)_{*}$. Also, call $\pi$ the natural isomorphism Aut $D \Im$ Aut $D^{(\mathfrak{p})}$ defined by $(\pi \gamma)\left(\alpha^{(\mathfrak{p})}\right)=(\gamma(\alpha))^{(\mathfrak{P})}, \alpha \in D, \gamma \in$ Aut D. From (1.3.1), (1.3.2) one sees that the following diagram is commutative:

$$
\begin{array}{ccc}
g\left(K / Z_{\mathbf{P}}\right) & \stackrel{\boldsymbol{\xi}_{\mathbf{P}}}{\longrightarrow} \text { Aut } D \\
\rho \downarrow & & \pi \downarrow \\
g\left(K^{(\mathbf{P})} / k^{(\mathfrak{p})}\right) & \stackrel{\eta}{\longrightarrow} \text { Aut } D^{(\mathfrak{p})} .
\end{array}
$$

Now, the field $N_{G(\mathcal{P}) / k}(\mathfrak{p})$ corresponds to $\operatorname{Ker} \eta$ and $N_{G / k}$ corresponds to $\operatorname{Ker} \xi$. On the other hand, $\operatorname{Ker} \eta=\rho\left(\operatorname{Ker} \xi_{\mathbf{P}}\right)=\rho\left(\operatorname{Ker} \xi \cap g\left(K / Z_{\mathbf{P}}\right)\right)$ corresponds to the residue field $\left(N_{G / k}\right)^{(\mathfrak{B})}$ where $\mathfrak{P}$ is the prime ideal of $N_{G / k}$ lying under $\mathbf{P}$ and hence we have $\left(N_{G / k}\right)^{\left(\mathfrak{B}_{1}\right)}=N_{\left.G^{(}\right) / k(\mathfrak{P})}$. Summarizing, we get the following

Proposition 1.3.3. Let $G$ be a connected semi-simple algebraic group defined over a number field $k$. Let $\mathfrak{p}$ be a prime ideal of $k$ and $\mathfrak{B}$ be a prime ideal of the nuclear field $N_{G / k}$ lying above $\mathfrak{p}$. Then, for almost all $\mathfrak{p}$, the residue field $\left(N_{G / k}\right)^{(\Re)}$ coincides with the nuclear field $N_{G^{\prime}}\left(\mathfrak{p}_{1 / k^{(\mathfrak{p})}}\right.$ for the group $G^{(\mathfrak{p})}$ obtained from $G$ by the reduction modulo $p$.

Rernark 1.3.4. By (1.2.3), (1.3.3), one can interpret the multiplicative structure of the order of the finite group $G_{k(\mathfrak{p})}^{(\mathfrak{p})}$ in terms of the law of decomposition of $p$ relative to $N_{G / k}$ over $k$ and thus attach to $G$ over $k$ a finite number of $L$-functions taken from the Galois extension $N_{G / k}$ of $k$. Details will be discussed in the next section. 


\section{The number $e(G / k)$}

Let $G$ be a connected semi-simple algebraic group defined over a number field $k$. Put

$$
e(G / k)=\Pi_{\mathfrak{p}}^{\prime} \frac{q^{\mathrm{dim} G}}{\left[G_{k(\mathfrak{p})}^{(\mathfrak{p})}\right]}, \quad q=N_{k} \mathfrak{p}
$$

where $\Pi^{\prime}$ means the product over almost all prime ideals $p$ in $k$ for which the reduced groups $G^{(\mathfrak{p})}$ remain connected and semi-simple. As for the convergence of the product, see [18, Appendix II]. This number $e(G / k)$ modulo $\mathbf{Q}^{*}$ is invariantly attached to $G$ over $k$. Let $G^{\prime}$ be another connected semi-simple algebraic group defined over $k$. Obviously one has

$$
e\left(G \times G^{\prime} / k\right) \sim e(G / k) e\left(G^{\prime} / k\right) .
$$

On the other hand, if $G^{\prime}$ is isogenous to $G$ over $k$, we get

$$
e(G / k) \sim e\left(G^{\prime} / k\right)
$$

because two isogenous groups over a finite field have the same number of rational points.

We say that a connected semi-simple algebraic group $G$ defined over $k$ is simple over $k$ ( $k$-simple, for short), if $G$ has no normal connected algebraic subgroup defined over $k$ except $G$ and $\{e\}$. It is clear that every semi-simple $G$ is isogenous to a product of $k$-simple groups $G_{i}, 1 \leqq i \leqq r$. From (1.4.1), (1.4.2), we get

$$
e(G / k) \sim e\left(G_{1} / k\right) \cdots e\left(G_{r} / k\right)
$$

Next, let $K$ be a finite extension of $k, H$ a connected semi-simple algebraic group defined over $K$ and let $G=R_{K / k}(H)$. One has then

$$
e(H / K) \sim e(G / k) .
$$

As for the proof, see e.g. [18, Appendix II].

Assume that $G$ is $k$-simple and let $H$ be any simple (i.e. $\bar{k}$-simple) factor of $G, K$ be the smallest field of definition for $H$ containing $k$. Then, one sees at once that $G$ is isogenous to the group $R_{K / k}(H)$ over $k$. By (1.4.3), (1.4.4), the computation of $e(G / k)$ is reduced to the case where $G$ is simple.

Let us assume that $G$ is simple. By (1.3.3) the residue field $\left(N_{G / k}\right)^{(\mathfrak{\beta})}$ is the nuclear field for $G^{(\mathfrak{p})}$ over $k^{(\mathfrak{p})}$ for almost all $\mathfrak{p}$, and so $e(G / k)$ can be ob- 
tained from Table 1. According to the structure of Aut $D$, we treat the following four cases separately.

Case i. $N_{G / k}=k$

Using the notation in (1.2.3), since $\sum_{\nu=1}^{l} a_{\nu}=l+N$, we get

$$
e(G / k) \sim \prod_{\nu=1}^{l} \zeta_{k}\left(a_{\nu}\right),
$$

where $\zeta_{k}$ is the zeta function of $k$. All groups of type $A_{1}, B_{l}(l \geqq 2), C_{l}(l \geqq 3)$, $E_{7}, E_{8}, F_{4}, G_{2}$ belong to this case.

Case 2. $\left[N_{G / k}: k\right]=2$

For this case, we denote by $L_{N / k}\left(N=N_{G / k}\right)$ the $L$-function with respect to the character belonging to the quadratic extension. This case can happen only for types $A_{l}(l \geqq 2), \quad D_{l}(l \geqq 4)$ and $E_{6}$.

TABLE 2

\begin{tabular}{c|c}
\hline type & \multicolumn{1}{c}{$e(G / k)\left(\bmod . \mathbf{Q}^{*}\right)$} \\
\hline$A_{l}(l \geqq 2)$ & $\zeta_{k}(2) L_{N / k}(3) \zeta_{k}(4) \ldots\left\{\begin{array}{l}\zeta_{k}(l+1): \\
L_{N / k}(l+1): \quad l \text { odd }\end{array}\right.$ \\
$D_{l}(l \geqq 4)$ & $L_{N / k}(l) \Pi_{\nu=1}^{l-1} \zeta_{k}(2 v)$ \\
$E_{6}$ & $\zeta_{k}(2) L_{N / k}(5) \zeta_{k}(6) \zeta_{k}(8) L_{N / k}(9) \zeta_{k}(12)$
\end{tabular}

Case 3. $\left[N_{G / k}: k\right]=3$

This case can happen only for the type $D_{4}$. We denote by $\chi$ the character belonging to the cubic extension which sends a generator of the Galois group to $\omega$, a primitive cubic root of 1 . As before we put $N=N_{G / k}$. From Table 1 we see that

$$
e(G / k) \sim L_{N / k}(\chi, 4) L_{N / k}(\bar{\chi}, 4) \zeta_{k}(2) \zeta_{k}(6) .
$$

By virtue of the relation $\zeta_{N}(4)=\zeta_{k}(4) L_{N / k}(\chi, 4) L_{N / k}(\bar{\chi}, 4)$, we get

$$
e(G / k) \sim \zeta_{N}(4) \zeta_{k}(4)^{-1} \zeta_{k}(2) \zeta_{k}(6) .
$$

Case 4. $\left[N_{G / k}: k\right]=6$

This case can happen only for the type $D_{4}$. We again put $N=N_{G / k}$. The Galois group $g(N / k)$ is isomorphic with $S_{3}$, the symmetric group on three letters. Let $M$ be the intermediate quadratic extension of $k$. Thus $N / M$ is a Galois cubic field. Let $\chi$ be the character of this cubic extension which sends a generator to $\omega$ as before and let $\chi^{*}$ be the character of $g(N / k)$ induced by 
$\chi$. Since $\chi^{*}$ takes values in $\mathbf{Q}$, we have $(\bar{\chi})^{*}=\chi^{*}$. From Table 1 , we see that

$$
e(G / k) \sim L_{N / k}\left(\chi^{*}, 4\right) \zeta_{k}(2) \zeta_{k}(6) .
$$

By a theorem on induced characters, we get

$$
L_{N / M}(\chi, 4)=L_{N / k}\left(\chi^{*}, 4\right)=L_{N / k}\left((\bar{\chi})^{*}, 4\right)=L_{N / M}(\bar{\chi}, 4) .
$$

On the other hand, we have

$$
\zeta_{N}(4)=\zeta_{M}(4) L_{N / M}(\chi, 4) L_{N / M}(\bar{\chi}, 4) .
$$

Hence, we get

$$
e(G / k) \sim \zeta_{N}(4)^{1 / 2} \zeta_{M}(4)^{-1 / 2} \zeta_{k}(2) \zeta_{k}(6) .
$$

\subsection{Property $(P)$}

We shall introduce in this section a property called $(P)$ for a connected semi-simple algebraic group defined over a number field $k$. As we shall see later on, this property will take two parts (topological and arithmetical) in our theory. We first recall some elementary facts.

Let $G$ be a connected real semi-simple Lie group with finite center, $g$ be the Lie algebra of $G$ and let $g^{\mathbf{C}}$ be the complexification of $g: g^{\mathbf{C}}=\mathbf{C} \otimes_{\mathbf{R}^{g}}$. By an involution of $g^{C}$ we mean an involutive sesquilinear automorphism \& of $g^{\mathrm{C}}$ with respect to $\mathbf{C} / \mathbf{R}$. We denote by $c_{0}$ the involution of $g^{\mathbf{C}}$ over $g$ given by ${ }_{\iota_{0}}(\lambda \otimes X)=\bar{\lambda} \otimes X$. For an involution ‘, put

$$
\mathfrak{u}_{\iota}=\left\{X \in g^{\mathbf{C}}, \iota(X)=X\right\},
$$

this being a real form of $g^{\mathrm{C}}$. An involution \& will be called compact if it satisfies the following two conditions:

$$
\iota_{0}=\iota_{0} s
$$

$\mathfrak{u}_{\iota}$ is a compact Lie algebra.

Denote by $\mathscr{C}$ the set of all compact involutions of $g^{\mathrm{C}}$. As is well known, $\mathscr{C}$ is non-empty. For an $\iota \in \mathscr{C}$, let $U_{\iota}$ be the connected compact Lie group whose Lie algebra is $\mathfrak{u}_{\iota}$ and let $K_{\iota}$ be the analytic subgroup of $G$ whose Lie algebra is $\mathfrak{f}_{\ell}=\mathfrak{g} \cap \mathfrak{u}_{\ell}$. The map $\rightarrow K_{\ell}$ gives a bijection of $\mathscr{C}$ with the set of all maximal compact subgroups of $G$. For two involutions $\iota, \iota^{\prime} \in \mathscr{C}$, one can find an element $g \in G$ such that

$$
\iota^{\prime}=A d(g)^{\mathbf{C}}\left(A d(g)^{\mathbf{C}}\right)^{-1}
$$


where $A d(g)^{\mathrm{C}}$ means the automorphism of $\mathrm{g}^{\mathrm{C}}$ obtained from $A d(g)$ by linearity. We have then

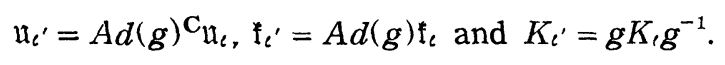

Fixing once for all a compact involution, of $\mathfrak{g}^{\mathrm{C}}$, put $\mathfrak{u}=\mathfrak{u}_{\iota}, \mathfrak{l}=\mathfrak{l}_{\iota}, U=U_{\iota}$, $K=K_{\iota}$. Also put,

$$
X=G / K, X_{u}=U / K \text {. }
$$

In view of (1.5.3), (1.5.4) all spaces $U, K, X, X_{\mathfrak{u}}$ are invariantly attached to $G$. For simplicity, we call $X$ the symmetric space attached to $G$ and call $U$ (resp. $X_{u}$ ) the dual of $G(\operatorname{resp}, X)$.

It is known that the Euler number $E\left(X_{u}\right)$ of the compact space $X_{u}$ is nonnegative and that $E\left(X_{u}\right)$ is positive if and only if $\operatorname{rank} U=\operatorname{rank} K([20$, p. 17]). When that is so, $\operatorname{dim} X_{u}(=\operatorname{dim} X)$ is even $([2$, p. 552 , Satz IV]).

Now, let $G$ be a connected semi-simple algebraic group defined over a number field $k$. Let $\infty_{i}, 1 \leqq i \leqq t$, be places at infinity of $k, k_{\infty_{i}}$ be the completion of $k$ with respect to $\infty_{i}$. Denote by $G_{i}$ the identity component of the topological group $G_{k_{\infty}}$. Viewing $G_{i}$ as a real semi-simple Lie group, we denote by $X_{i}$ the symmetric space attached to $G_{i}$ and by $\left(X_{i}\right)_{u}$ its dual. We shall say that $G$ is of type $(P)$ over $k$ if the Euler numbers $E\left(\left(X_{i}\right)_{u}\right)$ are positive for all $i, 1 \leqq i \leqq t$.

Proposition 1.5.5. If there is a connected semi-simple algebraic group $G$ defined over a number field $k$ over which $G$ is of type $(P)$, then $k$ is a totally real field.

In fact, suppose that $k$ is not totally real. Let $\infty_{1}$, say, be a complex place of $k: k_{\infty_{1}}=\mathbf{C}$. Then $G_{1}=G_{\mathrm{C}}$ is a complex Lie group. Denote by $g_{1}$ the complex Lie algebra of $G_{1}$ by $g_{0}$ the Lie algebra $g_{1}$ viewed as a real Lie algebra. Take any compact involution $\iota_{1}$ of $g_{1}$ and define an involution of $g_{0}^{\mathrm{C}}$ by $c(\lambda \otimes X)=$ $\bar{\lambda} \otimes \imath_{1}(X), X \in g_{0}$. One verifies at once the condition (1.5.1). On the other hand, one has, for $X, Y \in g_{0}$,

$$
(X+\sqrt{-1} \otimes Y)=\imath_{1}(X)-\sqrt{-1} \otimes \imath_{1}(Y),
$$

and hence $\mathfrak{n}_{\ell}=\mathfrak{a}_{\ell_{1}}+\sqrt{-1} \otimes \sqrt{-1} \mathfrak{l}_{\ell_{1}}$, which shows that $\mathfrak{u}_{\ell}$ is compact, i.e. 6 is compact (cf. (1.5.2)) and that $\mathfrak{f}_{\ell}=\mathfrak{u}_{\ell} \cap \mathfrak{g}_{0}=\mathfrak{u}_{\ell} \cap\left(\mathfrak{u}_{\ell_{1}}+\sqrt{-1} \mathfrak{u}_{\ell_{1}}\right)=\mathfrak{u}_{\ell_{1}}$. Denote by $t$ a maximal abelian subalgebra of $f_{\iota}$. Then, obviously, $t+\sqrt{-1} \otimes \sqrt{-1} t$ is an 
abelian subalgebra of $\mathfrak{u}_{\iota}$, and so rank $\mathfrak{u}_{\iota}>\operatorname{rank} \mathfrak{k}_{\iota}$, i.e. $E\left(\left(_{1}\right)_{u}\right)=0$, q.e.d.

Proposition 1.5.6. Groups $G$ and $G^{\prime}$ are of type $(P)$ over $k$ if and only if $G \times G^{\prime}$ is of type $(P)$ over $k$.

In fact, let $g_{i}, g_{i}^{\prime}$ be Lie algebras of $G_{k_{\infty i}}, G_{k_{\infty i}}^{\prime}$, respectively. Then $g_{i} \times g_{i}^{\prime}$ is the Lie algebra of $\left(G \times G^{\prime}\right)_{k_{\infty i}}$ and one can take a compact involution of $\left(g_{i} \times g_{i}^{\prime}\right)^{\mathbf{C}}$ which is the product of such involutions on each factor. Our assertion follows at once from the fact that the Euler number of the product space is the product of the Euler numbers of each space.

PROPOSITION 1.5.7. If $G^{\prime}$ is isogenous to $G$ over $k$ and if one of the groups is of type $(P)$ over $k$, then the other one is cf type $(P)$ over $k$.

In fact, using the notation in (1.5.6), an isogeny induces an isomorphism of Lie algebras $g_{i}^{\prime}$ with $\mathfrak{g}_{i}$, and so the "equal rank" property is invariant under the isogeny.

Proposition 1.5.8. Let $K$ be a finite extension of $k$ and let $H$ be a connected semi-simple algebraic group defined over $K$. Then, $H$ is of type $(P)$ over $K \Longleftrightarrow R_{K / k}(H)$ is of type $(P)$ over $k$.

In fact, for an infinite place $\infty$ of $k$, denote by $\infty_{i}, 1 \leqq i \leqq s$, be infinite places of $K$ lying above $\infty$. Then we have an isomorphism

$$
\left(R_{K / k}(H)\right)_{k_{\infty}} \cong \Pi_{i=1}^{s} H_{K_{\infty} i}
$$

$[25$, p. 9$]$, from this our assertion follows at once.

Corollary 1.5.9. Let $G$ be $k$-simple, let $H$ be any simple factor of $G$ and let $K$ be the smallest field of definition for $H$ containing $k$. If $G$ is of type $(P)$ over $k$, then $K$ is totally real.

In fact, $G$ is isogenous to $R_{K / k}(H)$ and so $H$ is of type $(P)$ over $K$ by (1.5.8). Our assertion then follows from (1.5.5).

\subsection{Chevalley basis}

Let $G$ be a connected semi-simple algebraic group defined over a field $k$ of characteristic zero and $T$ be a maximal torus of $G$ defined over $k$. Let $\mathfrak{g}, \mathfrak{h}$ be Lie algebras of $G, T$, respectively. If $G$ is imbedded in $G L(\boldsymbol{n})$, the Lie algebras $\mathfrak{g}, \mathfrak{h}$ are defined over $k$ as linear subvarieties of the ambient Lie algebra $\mathfrak{g l}(n)$. To each character $\xi \in \hat{T}=\operatorname{Hom}\left(T, G_{m}\right)$, there corresponds the 
differential $d \xi: \mathfrak{h} \rightarrow G_{a}$. If, in particular, $\alpha \in \hat{T}$ is a root in the sense of 1.1 , one verifies easily that $d \alpha$ becomes a root of $g$ with respect to the Cartan subalgebra $\mathfrak{h}$ in the ordinary sense ${ }^{*}$ and that the map $\alpha \rightarrow d \alpha$ gives a one-toone correspondence between the root systems in two senses. From now on, we shall identify these two root systems and denote again by $\Delta$ the set of all roots of $G$ (resp. g) with respect to $T$ (resp. $\mathfrak{h}$ ). For a root $\alpha \in \hat{T}$, it is easy to see that $\alpha^{\sigma}, \sigma \in g(\bar{k} / k)$, is again a root. Through the identification, one can define the action of $g(\bar{k} / k)$ on the infinitesimal roots by $(d \alpha)^{\sigma}=d \alpha^{\sigma}$. Thus $\mathfrak{g}(\bar{k} / k)$ permutes the elements in $\Delta$. Notice that if $X_{\alpha} \in \mathfrak{g}_{\bar{k}}$ is a root vector for $\alpha, X_{\alpha}^{\sigma}$ is a root vector for $\alpha^{\sigma}, \alpha \in \Delta, \sigma \in g(\bar{k} / k)$.

Let $B$ be the Killing form of $g$. For a root $\alpha \in \Delta$, we denote by $H_{\alpha}^{\prime}$ the element in $\mathfrak{h}_{\bar{k}}$ determined by the condition

$$
B\left(H_{\alpha}^{\prime}, H\right)=\alpha(H) \quad \text { for all } H \in \mathfrak{h} .
$$

Since $B$ is left fixed by $g(\bar{k} / k)$, i.e. $B\left(X^{\top}, Y^{\sigma}\right)=B(X, Y)^{\jmath}, X, Y \in g_{\bar{k}}, \sigma \in g(\bar{k} / k)$, we get

$$
H^{\prime} \alpha^{\sigma}=\left(H_{\alpha}^{\prime}\right)^{\sigma}, \quad \sigma \in g(\bar{k} / k) .
$$

From this we get

$$
\left\langle\alpha^{\sigma}, \beta^{\top}\right\rangle=\langle\alpha, \beta\rangle, \quad \alpha, \beta \in \Delta
$$

because $\langle\alpha, \beta\rangle\left(=B\left(H_{\alpha}^{\prime}, H_{\beta}^{\prime}\right)\right.$ by definition $)$ is a rational number. For $\alpha \in \Delta$, we shall put

$$
H_{\alpha}=\frac{2}{\langle\alpha, \alpha\rangle} H_{\alpha}^{\prime}
$$

From (1.6.1), (1.6.2), we get

$$
H_{\alpha^{\sigma}}=\left(H_{\alpha}\right)^{\sigma}, \quad \sigma \in \mathfrak{g}(\bar{k} / k) .
$$

Let $\Pi=\left\{\alpha_{1}, \ldots, \alpha_{l}\right\}$ be a system of fundamental roots. Then so is the transform $\Pi^{\sigma}=\left\{\alpha_{1}^{\sigma}, \ldots, \alpha_{l}^{\sigma}\right\}$. Since $H_{\alpha_{i}}, 1 \leqq i \leqq l$, form a for the module of co-weights $([9$, p. 16$])$ one can take $\left\{H_{x_{i}}\right\}_{1 \leqq i \leqq l}$ as a part of Chevalley basis. Thus, let

$$
\left\{H_{\alpha_{i}}, 1 \leqq i \leqq l, X_{\alpha}, \alpha \in \Delta\right\}
$$

* When $\alpha$ is given by (1.1.1), $X_{\alpha}=d x_{\alpha}$ (1) serves as a root vector in the sense of infinitesimal theory. 
be a Chevalley basis for $g_{\bar{k}}$, where $X_{\alpha}, \alpha \in \Delta$, are subject to the conditions described in [9, p. 24, Th. 1], i.e.

$$
\begin{aligned}
& {\left[X_{\alpha}, X_{-\alpha}\right]=H_{\alpha}, \quad \alpha \in \Delta} \\
& {\left[X_{\alpha}, X_{\beta}\right]=N_{\alpha, \beta} X_{\alpha+\beta} \text { if } \alpha, \beta, \alpha+\beta \in \Delta,}
\end{aligned}
$$

where $N_{\alpha, \beta}= \pm(p+1), p$ being the maximum of integers $i \geqq 0$ such that $\beta-i \alpha \in \Delta$.

Now, let $\mathbf{G}$ be a group of Chevalley type over $k$ and $T$ be a $k$-trivial maximal torus. Since $g(\bar{k} / k)$ acts on $\hat{T}$ trivially, one has $\alpha^{\sigma}=\alpha$ for all $\alpha \in \Delta$, $\sigma \in \mathfrak{g}(\bar{k} / k)$. Hence $H_{\alpha}, \alpha \in \Delta$, are left fixed by $g(\bar{k} / k)$ (cf. (1.6.4)). We shall next modify $X_{\alpha}, \alpha \in A$, so that all these are again left fixed by $\mathfrak{g}(\bar{k} / k)$. To do this, define numbers $\varepsilon_{\alpha}(\sigma), \alpha \in \Delta, \sigma \in g(\bar{k} / k)$, by

$$
\left(X_{\alpha}\right)^{\varsigma}=\varepsilon_{\alpha}(\sigma) X_{\alpha} .
$$

If we fix $\alpha, \varepsilon_{\alpha}(\sigma)$ satisfy

$$
\varepsilon_{\alpha}(\sigma \tau)=\varepsilon_{\alpha}(\sigma)^{\tau} \varepsilon_{\alpha}(\tau), \quad \sigma, \tau \in g(\bar{k} / k),
$$

i.e. $\left(\varepsilon_{\alpha}(\sigma)\right)$ is a cocycle of $g(\bar{k} / k)$ in $(\bar{k})^{*}$. On the other hand, if we fix $\sigma$, we see from (1.6.4), (1.6.5) that

$$
\begin{array}{ll}
\varepsilon_{-\alpha}(\sigma)=\varepsilon_{\alpha}(\sigma)^{-1} & \text { for } \alpha \in \Delta, \\
\varepsilon_{\alpha+\beta}(\sigma)=\varepsilon_{\alpha}(\sigma) \varepsilon_{\beta}(\sigma) & \text { if } \alpha, \beta, \alpha+\beta \in \Delta .
\end{array}
$$

Let $\Pi=\left\{\alpha_{1}, \ldots, \alpha_{l}\right\}$ be a system of fundamental roots and let $\alpha=\sum_{i=1}^{l} m_{i} \alpha_{i}$ be the unique expression of $\alpha \in \Delta$ with $m_{i} \in \mathbf{Z}$. For each $\alpha_{i}$, let $a_{\alpha_{i}}$ be a solution of (1.6.6), i.e. $\varepsilon_{\alpha_{i}}(\sigma)=a_{\alpha_{i}}^{1-\sigma}$, and put $a_{\alpha}=\prod_{i=1}^{l} a_{\alpha_{i}}^{m_{i}}$ when $\alpha=\sum_{i=1}^{l} m_{i} \alpha_{i}$. Then, from (1.6.7) we see that $\boldsymbol{a}_{\alpha}$ is a solution of (1.6.6): $\varepsilon_{\alpha}(\sigma)=a_{\alpha}^{1-\sigma}$. Hence $Y_{\alpha}=a_{\alpha} X_{\alpha}$ are left fixed by $g(\bar{k} / k)$. Obviously, $Y_{\alpha}, \alpha \in \Lambda$, satisfy (1.6.5). Therefore the existence of Chevalley basis which is invariant under $g(\bar{k} / k)$ is settled.

From now on, whenever $\mathbf{G}$ is of Chevalley type over $k$, we shall understand by $a$ Chevalley basis of the Lie algebra $g$ of $\mathbf{G}$ a Chevalley basis in the usual sense which is already a basis for $\mathrm{g}_{k}$.

We next want to associate a compact involution to a Chevalley basis. Taking $\mathbf{C}$ as a universal domain, let $G$ be a connected semi-simple algebraic group defined over $\mathbf{R}, g$ be the Lie algebra of $G$ and let $\left\{H_{\alpha_{i}}, 1 \leqq i \leqq l, X_{\alpha}, \alpha \in \Delta\right\}$ 
be a Chevalley basis of $g$ with respect to a Cartan subalgebra $\mathfrak{h}$ of $\mathfrak{g}$ defined over R. Obviously $g$ is the complexification of $g_{R}$ and we denote as before by $s_{0}$ the involution of $\mathfrak{g}$ over $\mathfrak{g}_{\mathbf{R}}: \iota_{0}(X)=\bar{X}, X \in \mathfrak{g}$. Define $u_{\alpha}$ by the relation $\iota_{0}\left(X_{\alpha}\right)=\bar{X}_{\alpha}=u_{\alpha} X_{\bar{\alpha}}, \alpha \in \Delta$, and put

$$
\text { c( } \left.H_{\alpha_{i}}\right)=-H_{\alpha_{i}}, \quad \iota\left(X_{\alpha}\right)=-\left|u_{\alpha}\right| X_{-\alpha} .
$$

One then easily verify that $c$ is a compact involution of $g$. We shall call this involution the compact involution of $\mathrm{g}$ associated with a Chevalley basis $\left\{H_{\alpha_{i}}, X_{\alpha}\right\}$ of g. If, in particular, $\mathbf{G}$ is of Chevalley type over $\mathbf{R}$ and $\left\{H_{q_{i}}, X_{\alpha}\right\}$ is a Chevalley basis (in the strict sense above) for the Lie algebra $\mathbf{g}$ of $\mathbf{G}$, then $u_{\alpha}=1, \alpha \in \Delta$, because the Chevalley basis is already a basis for $\mathbf{g}_{\mathbf{R}}$, and the associated involution is given by

$$
\iota\left(H_{\alpha_{i}}\right)=-H_{\alpha_{i}}, \quad \iota\left(X_{\alpha}\right)=-X_{-\alpha} .
$$

For a complex semi-simple Lie algebra $\mathfrak{g}$, we denote by Int $g$ the identity component of the topological group Aut 8 . Now, assume that $\mathbf{G}$ is a group of Chevalley type over $\mathbf{R}$ which is simple. Let $\iota_{0}$, , be involutions of $\mathbf{g}$ defined above. Then $A=c_{0} c=c_{0}$ is an automorphism of $\mathrm{g}$. We shall ask the question: whether $A \in \operatorname{Int} \mathrm{g}$ or $A \notin \operatorname{Int} \mathrm{g}$ ?. In view of the structure of Aut $\mathrm{g} / \mathrm{Int} \mathrm{g}$ it is enough to consider the cases $A_{l}(l \geqq 2), D_{l}(l \geqq 4)$ and $E_{6}$. For these cases, all roots have the same length and from (1.6.3), (1.6.9), we get

$$
A\left(H_{\alpha_{i}}\right)=H_{-\alpha_{i}}, \quad A\left(X_{\alpha}\right)^{*}=-X_{-\alpha} .
$$

First of all, we see at once that $\operatorname{det} A=(-1)^{l+N}$, where $N$ denotes the number of positive roots. If $\mathrm{g}$ is of type $D_{l}(l:$ odd $\geqq 5)$, then $l+N=l^{2}$ is odd, and so $A \notin$ Int $\mathbf{g}$. Next, we consider the case $E_{6}$. Since $A$ fixes $\mathbf{h}=\left\{H_{\alpha_{i}}\right\}$ as a whole, $A$ induces a linear transformation $A^{*}$ of $\mathbf{h}^{*}=\operatorname{Hom}(\mathbf{h}, \mathbf{C})$ given by $A^{*}(\lambda)(H)$ $=\lambda(A(H)), H \in \mathbf{h}, \lambda \in \mathbf{h}^{*}$. From (1.6.10) we see that $\left(A^{-1}\right)^{*}(\alpha)=-\alpha$ for all $\alpha \in \Delta$. Now, if $A$ is in Int $\mathrm{g},\left(A^{-1}\right)^{*}$ must be contained in the Weyl group. However, it is known that in the case $\mathrm{E}_{0}$ there is no element $w$ in the Weyl group such that $w(\alpha)=-\alpha$ for all $\alpha \in \Delta$ (See [10, 19-08, line 12 from the bottom]). Thus we have $A \notin$ Int $\mathbf{g}$ for $E_{6}$. Thirdly, we consider the case $A_{l}$ $(l \geqq 2)$, Since $\mathrm{g}$ is isomorphic to $\operatorname{gl}(l+1)$, by taking the standard Chevalley basis of $g_{l}(l+1)$ (see e.g. [19]) we see that $A(X)=-{ }^{t} X, X \in \mathrm{g}$, and hence $A \notin$ Int $\mathrm{g}$. Lastly, let $\mathrm{g}$ be of type $D_{l}(l$; even $\geqq 4)$. We may put 


$$
\mathrm{g}=\left\{X \in g\left((2 l),{ }^{t} X S+S X=0\right\}, \quad S=\left(\begin{array}{cc}
0 & I_{l} \\
I_{l} & 0
\end{array}\right)\right.
$$

Then, by taking the standard Chevalley basis (see [19]), we see that $A(X)=-{ }^{t} X$, $X \in \mathrm{g}$. Hence, $A(X)=-{ }^{t} X=\mathrm{S} X \mathrm{~S}^{-1}$. However, one has $S \in \mathrm{SO}(\mathrm{S})$ because ${ }^{t} \mathrm{SSS}=S$ and $l$ is even. Hence $A(X)=A d(S) X$ and so $A \in \operatorname{Int} \mathrm{g}$. Summarizing, we get the following

$\mathrm{P}_{\mathrm{RO} O \mathrm{pition}}$ 1.6.11. Let $\mathbf{G}$ be a simple group of Chevalley type over $\mathbf{R}$, let $t_{0}$ be the involution of the Lie algebra $\mathrm{g}$ of $\mathbf{G}$ over $\mathrm{g}_{\mathrm{R}}$ and let " be the compact involution of $\mathrm{g}$ associated with a Chevalley basis of $\mathrm{g}$. Then the automorphism $A=c_{0} \iota=\iota_{1}$ is not contained in Int $\mathrm{g}$ if and only if $\mathrm{g}$ is one of types $A_{l}(l \geqq 2)$, $D_{l}(l:$ odd $\geqq 5)$ or $E_{6}$.

\subsection{Effect of $(P)$ on $N_{G / k}$}

We first consider algebraic groups over $\mathbf{R}$. Let $G$ be a connected semisimple algebraic group defined over $\mathbf{R}$ and let $\phi$ be an isomorphism, defined over $\mathbf{C}$, of $G$ with a group $\mathbf{G}$ of Chevalley type over $\mathbf{R}$. We see that $f=\bar{\phi} \phi^{-1}$ is an automorphism of $\mathbf{G}$ such that $\bar{f} f=$ id. Let $\mathfrak{g}, \mathbf{g}$ be Lie algebras of $G, \mathbf{G}$, respectively. Since $\overline{d \phi}=d \bar{\phi}$, we see that $d f=\overline{d \phi}(d \phi)^{-1}$ and that $\overline{d f} d f=$ id.. For simplicity, we put $d f=F$, this being an automorphism of $\mathrm{g}$. As before, we denote by $s_{0}$ the involution of $\mathrm{g}$ over $\mathrm{g}_{\mathbf{R}}:{ }_{c_{0}}(X)=\bar{X}, X \in \mathrm{g}$, by 6 the compact involution of $\mathrm{g}$ associated with a Chevalley basis and put $A=\varepsilon_{0} \varepsilon=\iota_{0}$. To the equality

$$
\phi\left(G_{\mathbf{R}}\right)=\{x \in \mathbf{G}, \overline{f(x)}=x\}
$$

corresponds the equality as Lie algebras over $\mathbf{R}$ :

$$
d \phi\left(\mathfrak{g}_{\mathrm{R}}\right)=\{X \in \mathrm{g}, \overline{F(\bar{X})}=X\} .
$$

From now on, we put $g_{1}=d \phi\left(g_{\mathrm{R}}\right)$, this being a real form of $\mathrm{g}$. If we define an involution $\iota_{1}$ of $g$ by $\iota_{1}=c_{0} F, g_{1}$ is nothing but the set of fixed points by $\iota_{1}$. Furthermore, denote by $\iota^{*}$ a compact involution of $\mathrm{g}=g_{1}^{\mathbf{C}}$. By definition, $\iota^{*}$ satisfies the following conditions:

$\iota_{1} \iota^{*}=\iota^{*} \iota_{1}, \mathfrak{u}^{*}=\left\{X \in \mathrm{g}, \iota^{*}(X)=X\right\}$ is compact. We put $A_{1}=\imath_{1} \iota^{*}=\iota^{*} \iota_{1} \in$ Aut $\mathrm{g}$. Since involutions $\iota, c^{*}$ of $\mathbf{g}$ are both compact, there is a $J$ in Int $\mathbf{g}$ such that $\iota=J \iota^{*}([11$, p. 158, Cor. 7.3$])$. We get then the relation:

$$
A=\ell_{0} \iota=\left(\ell_{0} \iota_{1}\right)\left(\iota_{1} \iota^{*}\right)\left(\iota^{*} \iota\right)=F A_{1} J, \quad J \in \text { Int g. }
$$


Now, put $G_{\eta}=\left(G_{\mathbf{R}}\right)_{0}$, the topological identity component of $G_{\mathbf{R}}$, and assume that

The Euler number $E\left(X_{u}\right)>0$,

where $X_{u}$ is the compact space dual to the symmetric space attached to $G_{0}$. Since $g_{R} \cong g_{1}$ as real Lie algebras, the assumption $(\#)$ is equivalent to say that

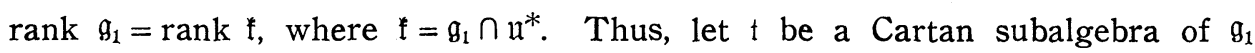
which is also contained in $\mathfrak{f}$. Since $A_{1}$ leaves $\mathfrak{f}$ elementwise fixed, it leaves $\mathrm{t}^{\mathrm{C}}$, a Cartan subalgebra of $\mathrm{g}$, elementwise fixed and so $A_{1}$ is contained in Int $\mathrm{g}$ ([14, 16-03, Prop. 1]). Hence, under (\#), we see from (1.7.1) that

$$
A \in \text { Int } \mathrm{g} \Longleftrightarrow F \in \text { Int } \mathrm{g} \text {. }
$$

Now, let $N_{G / \mathbf{R}}$ be the nuclear field for $G$ over $\mathbf{R}$, this being either $\mathbf{R}$ or $\mathbf{C}$. Obviously, we have

$$
N_{a / \mathbf{R}}=\mathbf{R} \Longleftrightarrow f \in \operatorname{Inn} \mathbf{G} .
$$

However, since we have $f \in \operatorname{Inn} \mathbf{G} \Longleftrightarrow F=d f \in \operatorname{Int} \mathbf{g}$, we get from (1.7.2) the following relation

$$
N_{G / \mathbf{R}}=\mathbf{R} \Longleftrightarrow A \in \operatorname{Int} \mathbf{g} \quad \text { (under }(\#) \text { ). }
$$

Combining (1.7.4) with (1.6.11), we get

Proposition 1.7.5. Let $G$ be a connected simple algebraic group defined over $\mathbf{R}$ satisfying the condition (\#). Then $N_{G / \mathbf{R}}=\mathbf{C}$ if and only if $G$ is one of types $A_{l}(l \geqq 2), D_{l}(l:$ odd $\geqq 5)$ or $E_{6}$.

We now come back to our original situation: let $k$ be a number field and let $G$ be a $k$-simple algebraic group defined over $k$. Since all simple factors of $G$ are of the same type, we may mention the type of $G$. The following is a main result of this section.

THEOREM 1.7.6. Let $G$ be a k-simple algebraic group over a number field $k, N_{G / k}$ be the nuclear field for $G$ over $k$. Assume that $G$ is of type $(P)$ over $k$. Then $k$ is totally real and $N_{\alpha / k}$ is totally real or totally imaginary. More precisely, we have

$N_{a / k}$ is totally imaginary $\Longleftrightarrow G$ is of type $A_{l}(l \geqq 2), D(l:$ odd $\geqq 5)$

$$
\text { or } E_{6} \text {. }
$$


Proof. That $k$ is totally real follows from (1.5.9). Next, let $H$ be any simple factor of $G$ and $K$ be the smallest field of definition for $H$ containing $k$. Hence $G$ is isogenous to $R_{K / k}(H)$. In view of (1.1.4), (1.1.6), (1.5.8), we may assume without loss of generality that $G$ is already $\bar{k}$-simple. Let $\infty_{i}$, $1 \leqq i \leqq t$, be all infinite places of $k$. Then the group $G$ viewed as an algebraic group defined over $k_{s_{i}}(\cong \mathbf{R})$ satisfies the condition $(\#)$ for all $i, 1 \leqq i \leqq t$. Hence, by (1.7.5), the reality or the imaginarity of $N_{G / k \infty_{i}}$ is independent of $i$, and depends only upon the type of $G$. From (1.1.5) we have $N_{G / k_{\infty i}}=k_{\infty_{i}} N_{G / k}$. Therefore $N_{G / k}$ is totally real or totally imaginary. The rest of the theorem follows from (1.7.5), q.e.d.

\section{Discriminant}

\subsection{The number $\mu(G / k)$}

Let $k$ be a field of characteristic zero and let $G$ be a connected semi-simple algebraic group defined over $k$. Let $\phi$ be an isomorphism defined over $\bar{k}$ of $G$ with a group $\mathbf{G}$ of Chevalley type over $k$. Put $f_{\sigma}=\phi^{\sigma} \phi^{-1}, \sigma \in g(\bar{k} / k)$. Then $d f_{\sigma}=d \phi^{\sigma} d \phi^{-1}$ is an automorphism of the Lie algebra $\mathbf{g}$ of $\mathbf{G}$. Suppose that $\phi^{\prime}$ is another isomorphism of $G$ with a group $\mathbf{G}^{\prime}$ of Chevalley type over $k$. Let $f_{\sigma}^{\prime}=\phi^{\prime \pi} \phi^{\prime-1}$ and let $\psi$ be an isomorphism of $\mathbf{G}^{\prime}$ with $\mathbf{G}$ such that $\psi \phi^{\prime}=\phi$. Since $\psi^{\sigma} \psi^{-1}$ is in Inn $\mathbf{G}$ by (1.1.2), we see at once that $\operatorname{det}\left(d f_{\sigma}\right)=\operatorname{det}\left(d f_{\sigma}^{\prime}\right)$. Now, $\left(d f_{\sigma}\right)$ is a cocycle of $g(\bar{k} / k)$ in Aut $\mathbf{g}$ and $\operatorname{det}\left(d f_{\sigma}\right)= \pm 1$, we get a homomorphism $\sigma \rightarrow \operatorname{det}\left(d f_{\sigma}\right)$ of $g(\bar{k} / k)$ into the group $\{ \pm 1\}$, which is invariantly attached to $G$ over $k$. We shall denote by $M_{G / k}$ the extension of $k$ which corresponds to the kernel of $\sigma \rightarrow \operatorname{det}\left(d f_{\sigma}\right)$. Obviously, $M_{G / k}$ is at most quadratic over $k$. We denote by $\mu(G / k)$ a solution of the cocycle $\left(\operatorname{det}\left(d f_{\sigma}\right)\right)$ :

$$
\operatorname{det}\left(d f_{\sigma}\right)=\mu(G / k)^{\sigma-1} \text {. }
$$

Hence we have

$$
M_{G / k}=k(\mu(G / k)) .
$$

Needless to say, $\mu\left(G / k^{\prime}\right)$ modulo $k^{*}$ is an invariant of $G$ over $k$. If $G^{\prime}$ is another connected semi-simple algebraic group defined over $k$, one has clearly

$$
\mu\left(G \times G^{\prime} / k\right) \equiv \mu(G / k) \mu\left(G^{\prime} / k\right) \quad \bmod . k^{*} .
$$

On the other hand, if $G^{\prime}$ is isogenous to $G$ over $k$, we have 


$$
\mu(G / k) \equiv \mu\left(G^{\prime} / k\right) \quad \bmod . k^{*},
$$

because $\mu(G / k)$ depends only upon infinitesimal data.

Proposition 2.1.3. Notation being as above, let $N_{G / k}$ be the nuclear field for $G$ over $k$. Then $M_{G / k}$ is a subfield of $N_{G / k}$.

In fact, if a $\sigma \in g(\bar{k} / k)$ is in the group $\mathfrak{n}_{G / k}$ corresponding to $N_{G / k}$, then one has $f_{o} \in \operatorname{Inn} \mathbf{G}$, hence $d f_{o} \in \operatorname{Int} \mathbf{g}$ and so $\operatorname{det}\left(d f_{o}\right)=1$. q.e.d.

Corollary 2.1.4. If $\left[N_{G / k}: k\right]$ is odd, then $\mu(G / k) \in k^{*}$.

In fact, $M_{G / k}$ is at most quadratic extension of $k$, q.e.d.

Remark 2.1.5. Suppose that $G$ is simple. Then, one has $\mu(G / k) \in k^{*}$ for groups of type $A_{1}, B_{l}(l \geqq 2), C_{l}(l \geqq 3), E_{7}, E_{8}, F_{4}, G_{2}$, because $\left[N_{G / k}: k\right]=1$. If $G$ is of type $D_{4}$ and $\left[N_{G / k}: k\right]=3$, one also has $\mu(G / k) \in k^{*}$.

We shall next give another interpretation of the map $\sigma \rightarrow \operatorname{det}\left(d f_{o}\right)$. In general, let $f$ be an automorphism of a connected semi-simple algebraic group $G$ in characteristic zero. In 1.1 , we associated with $f$ an automorphism $f_{*} \in$ Aut $D, D$ being the Dynkin diagram with respect to a maximal torus $T$ and a Borel subgroup containing $T$. We have first defined $f_{*}$ for such an $f$ that $f(T)=T, f(B)=B$, and put $f_{*}=(\hat{f})^{-1}$, where $\hat{f}: \hat{T} \rightarrow \hat{T}$ is the dual of $f$ restricted on $T$. Let $\mathfrak{h}$ be the Lie algebra of $T$. The conditions $f(T)=1$, $f(B)=B$ imply that $d f$ induces an automorphism of the infinitesimal Dynkin diagram $d D$ of the Lie algebra of $G$ in the same way as $f$ induces $f_{*}$. Let us denote the induced automorphism of $d D$ by $(d f)_{*}$, which can, of course, be defined for any $f \in$ Aut $G$. It is clear that the identification of $D$ with $d D$ under $\alpha \rightarrow d \alpha$ (cf. the beginning of 1.6 ) identifies $f_{*}$ with $(d f)_{*}$. We shall denote by $\operatorname{sign} f_{*}$ the sign of $f_{*}$ as a permutation on $D$.

Proposition 2.1.6. Using the above notation, one has

$$
\operatorname{det}(d f)=\operatorname{sign} f_{*} \quad \text { for } f \in \text { Aut } G .
$$

In fact, without loss of generality, we may assume that $f(T)=T, f(B)=B$. Let $\Pi=\left\{\alpha_{1}, \ldots, \alpha_{l}\right\}$ be the system of fundamental roots relative to $T, B$, and let $\left\{H_{\alpha_{i}}, 1 \leqq i \leqq l, X_{\alpha}, \alpha \in \Delta\right\}$ be a Chevalley basis of the Lie algebra $g$ of $G$ with respect to the Lie algebra $\mathfrak{h}$ of $T$. We have then

$$
d f\left(H_{\alpha_{i}}\right)=H_{f *\left(\alpha_{i}\right)}, \quad 1 \leqq i \leqq l,
$$




$$
d f\left(X_{\alpha}\right)=u_{\alpha} X_{f *(\alpha)} \text { with } u_{\alpha} u_{-\alpha}=1, \quad \alpha \in \Delta .
$$

Let $\mathfrak{g}=\mathfrak{h})+\sum_{\alpha>0} \mathfrak{g}^{\alpha}+\sum_{\alpha>0} \mathfrak{g}^{-\alpha}$ be the decomposition of $\mathfrak{g}$ into eigen spaces $(\alpha>0$ means that $\alpha=\sum_{i=1}^{l} m_{i} \alpha_{i}$ with $m_{i} \geqq 0$ ). By the assumption, the automorphism $d f$ leaves each of $\mathfrak{h}, \mathfrak{y}^{+}=\sum_{\alpha>0} \mathfrak{g}^{\alpha}, \mathfrak{g}^{-}=\sum_{\alpha>0} g^{-\alpha}$ invariant. Accordingly, we have

$$
\operatorname{det}(d f)=\operatorname{det}(d f \mid \mathfrak{h}) \operatorname{det}\left(d f \mid \mathfrak{g}^{+}\right) \operatorname{det}\left(d f \mid \mathfrak{g}^{-}\right),
$$

where we see at once that $\operatorname{det}\left(d f \mid g^{+}\right) \operatorname{det}\left(d f \mid g^{-}\right)=1$ from (2.1.8), and our assertion follows from (2.1.7).

Proposition 2.1.9. Using the above notation, we have

$$
\mu(G / k) \in k^{*} \Longleftrightarrow\left(f_{0}\right)_{*} \text { are even permutations for all } \sigma \in g(\bar{k} / k) .
$$

In fact, we have

$$
\begin{aligned}
\mu(G / k) \in k^{*} & \Leftrightarrow \operatorname{det}\left(d f_{o}\right)=1 \text { for all } \sigma \in g(\bar{k} / k) \\
& \Leftrightarrow \operatorname{sign}\left(f_{o}\right)_{*}=1 \text { for all } \sigma \in g(\bar{k} / k) .
\end{aligned}
$$

Remark 2.1.10. Suppose that $G$ is simple. One can then determine the field $M_{i / k}$ by looking at the Dynkin diagram. In view of (2.1.4), it is enough to consider the case where $\left[N_{G / k}: k\right]$ is even.

Case (i) $\left[N_{G / k}: k\right]=2$

In this case, the only non-trivial $\left(f_{o}\right)_{*}$ comes from the non-trivial automorphism of the quadratic extension.

Type $A_{l}(l \geqq 2)$. We have

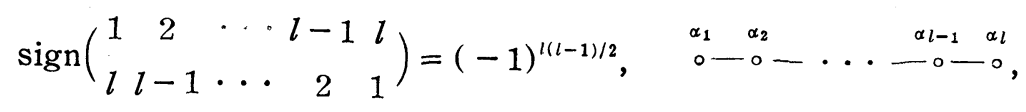

and so $M_{G / k}=k$ or $N_{G / k}$ according as $l \equiv 0,1$ or $l \equiv 2,3(\bmod .4)$.

Type $D_{l}(l \geqq 4)$. We have

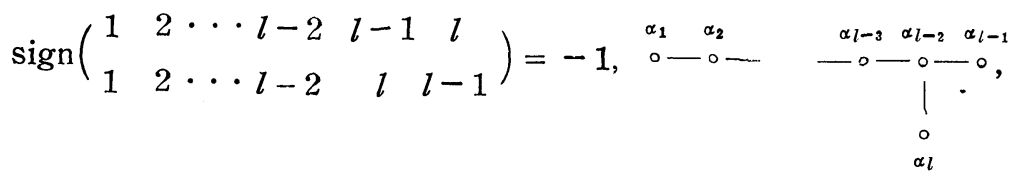

and so $M_{G / k}=N_{G} / k$. 
Type $E_{6}$. We have

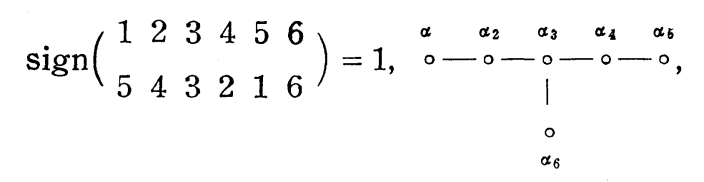

and so $M_{\theta / k}=k$.

Case (ii) $\left[N_{G / k}: k\right]=6$

This happens for $D_{4}$. Since the Galois groupe of $N_{G / k}$ over $k$ is isomorphic to Aut $D \cong S_{3}$, some $\left(f_{\sigma}\right)_{*}$ is an odd permutation. Hence, from (2.1.9), we see that $M_{G / k}$ is the unique quadratic subextension of $N_{G / k}$.

We shall next give one more interpretation of the

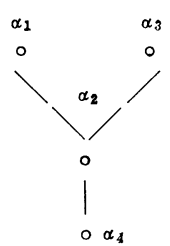
number $\mu(G / k)$ which will be used in the analytical consideration. Let $\mu(G / k)$ which will be used in the analytical consideration. Let $G, \phi, \mathbf{G}, g, g$ be as before (see the beginning of 2.1 ), let

$$
\left\{H_{\alpha_{i}}, 1 \leqq i \leqq l, X_{\alpha}, \alpha \in \Delta\right\}
$$

be a Chevalley basis for $\mathbf{g}$ with respect to a Cartan subalgebra of $\mathbf{g}$ defined over $k$. For simplicity, we put $\psi=d \phi, F_{\sigma}=\phi^{\top} \psi^{-1}\left(=d f_{o}\right), \sigma \in g(\bar{k} / k)$. We shall often denote by $\left\{Y_{1}, \ldots, Y_{d}\right\}, d=\operatorname{dim} \mathrm{g}$, the basis (2.1.11). Since $\left(F_{o}\right)$ is a cocycle, by Hilbert theorem 90 , there is a matrix $M \in G L(d)_{\bar{k}}$ such that

$$
\left(F_{\sigma}\left(Y_{1}\right), \ldots, F_{\sigma}\left(Y_{d}\right)\right)=\left(Y_{1}, \ldots, Y_{d}\right) M^{\sigma} M^{-1} .
$$

Since $\operatorname{det} F_{\sigma}=\operatorname{det}\left(d f_{\sigma}\right)$, we have

$$
\mu(G / k) \equiv \operatorname{det} M \quad \bmod . k^{*} \text {. }
$$

Using this $M$, define a basis $\left\{X_{1}, \ldots, X_{d}\right\}$ of $\mathrm{g}$ by the relation

$$
\left(\phi\left(X_{1}\right), \ldots, \phi\left(X_{d}\right)\right)=\left(Y_{1}, \ldots, Y_{d}\right) M .
$$

It is easy to see that $X_{i}^{\sigma}=X_{i}$, for all $i$ and $\sigma$, i.e. $\left\{X_{i}\right\}$ is already a basis for $\mathrm{g}_{k}$. Let $B, \mathbf{B}$ be Killing forms of $\mathrm{g}$. $\mathrm{g}$, respectively. Clearly, one has $B(X, Y)$ $=\mathbf{B}(\psi X, \psi Y), X, Y \in \mathfrak{g}$. Hence, from (2.1.14) we get

$$
\operatorname{det}\left(B\left(X_{i}, X_{j}\right)\right)=\operatorname{det}\left(\mathbf{B}\left(Y_{i}, Y_{j}\right)\right)(\operatorname{det} M)^{2} .
$$

Let $\Delta^{+}$(resp. $\Delta^{-}$) be the set of positive (resp. negative) roots of $\mathrm{g}$ determined by $\Pi=\left\{\alpha_{1}, \ldots, \alpha_{l}\right\}$ and let $N$ be the number of elements in $\Delta^{+}$. 
Arranging $\left\{Y_{1}, \ldots, Y_{d}\right\}$ in the order $\left\{H_{\alpha_{i}}, X_{\alpha}, X_{-\alpha}, \ldots\right\}$ we see that the matrix $\left(\mathbf{B}\left(Y_{i}, Y_{j}\right)\right)$ has the form

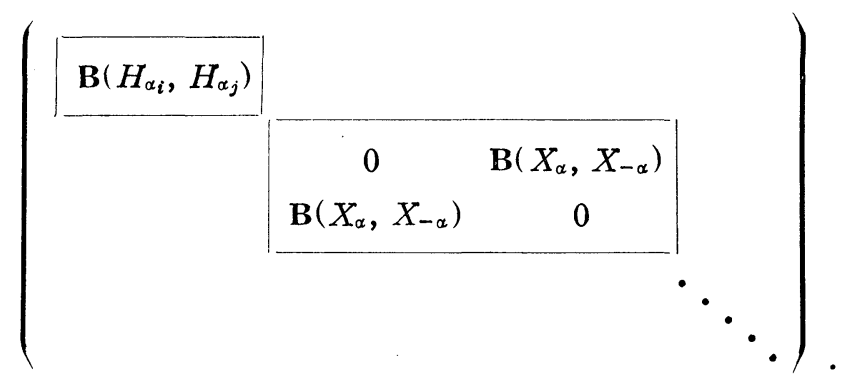

A simple computation then show that

$$
\operatorname{det}\left(\mathbf{B}\left(Y_{i}, Y_{j}\right)\right)=(-1)^{N} 2^{d} C \Pi_{i=1}^{l}\left\langle\alpha_{i}, \alpha_{i}\right\rangle^{-1} \Pi_{\alpha>0}\langle\alpha, \alpha\rangle^{-2},
$$

where $C=\operatorname{det}\left(\frac{2\left\langle\alpha_{i}, \alpha_{j}\right\rangle}{\left\langle\alpha_{j}, \alpha_{j}\right\rangle}\right)$ is a positive integer. The right hand side of (2.1.16) is a rational number which depends only upon the type of g. From now on, we shall put

$$
q(g)=(-1)^{N} 2^{d} C \Pi_{i=1}^{l}\left\langle\alpha_{i}, \alpha_{i}\right\rangle^{-1} \Pi_{\alpha>0}\langle\alpha, \alpha\rangle^{-2}
$$

for a semi-simple Lie algebra $g$ in characteristic zero. Clearly, one has $q\left(g \times g^{\prime}\right)$ $=q(g) q\left(g^{\prime}\right)$ where $g^{\prime}$ is another semi-simple Lie algebra. If we take arbitrary basis $\left\{X_{i}^{\prime}\right\}$ of $g_{k}$, we have

$$
\operatorname{det}\left(B\left(X_{i}^{\prime}, X_{j}^{\prime}\right)\right) \equiv \operatorname{det}\left(B\left(X_{i}, X_{j}\right)\right) \quad \bmod .\left(k^{*}\right)^{2} .
$$

Hence, from (2.1.13), (2.1.15) we get

Proposition 2.1.18. Let $\left\{X_{i}\right\}$ be any basis of $\mathrm{g}_{k}$, then we have

$$
\operatorname{det}\left(B\left(X_{i}, X_{j}\right)\right) \equiv q(\mathfrak{g}) \mu(G / k)^{2} \quad \bmod .\left(k^{*}\right)^{2} .
$$

In other words, one can define $\mu(G / k)$ by

$$
\mu(G / k) \equiv\left(\operatorname{det}\left(B\left(X_{i}, X_{j}\right)\right) q(\mathrm{~g})^{-1}\right)^{1 / 2} \quad \text { mod. } k^{*} .
$$

We now want to consider the effect of $R_{K / k}$. Let $K$ be a finite extension of $k, H$ be a connected semi-simple algebraic group defined over $K$ and let $\mathfrak{h}$ be the Lie algebra of $H$. Let

$$
g(\bar{k} / k)=g(\bar{k} / K)_{\sigma_{1}}+\cdots+g(\bar{k} / K)_{\sigma_{\delta}}, \quad \delta=[K: k],
$$

be the right cosets decomposition where we put $\sigma_{1}=\mathrm{id}$. Let $\left\{X_{i}\right\}_{1 \leqq i \leqq d}$ be a 
basis of $\mathfrak{h}_{K}$. Then $\left\{X_{i}^{\sigma \nu}\right\}$ will be a basis of $\mathfrak{h}_{K}^{\sigma_{\nu} \sigma_{\nu}}$, where $\mathfrak{h}^{\sigma_{\nu}}$ is the Lie algebra of $H^{\sigma_{\nu}}$ which is defined over $K^{\sigma_{\nu}}$. Since $q(\mathfrak{h})=q\left(\mathfrak{h}^{\sigma_{\nu}}\right)$, we see from (2.1.19) that

$$
\mu(H / K)^{\sigma_{\nu}}= \pm a^{\sigma_{\nu}} \operatorname{det}\left(B\left(X_{i}, X_{j}\right)^{\sigma_{\nu}}\right)^{1 / 2} q(\mathfrak{h})^{-1 / 2}, \quad a \in K^{*}, 1 \leqq \nu \leqq \delta,
$$

where $B$ being the Killing form of $\mathfrak{h}$. Put $G=R_{K / k}(H)$. By definition, there is a map $p: G \rightarrow H$, defined over $K$, such that $G$ is isomorphic over $\bar{k}$ with the product $H^{\sigma_{1}} \times \cdots \times H^{\sigma_{\delta}}$ under the map $\pi=\left(p^{\sigma_{\nu}}\right)_{1 \leqq \nu \leqq \delta}$. We shall again denote by $\pi=\left(p^{\sigma_{\nu}}\right)$ the isomorphism of the Lie algebra $g=R_{K / k}(\mathfrak{h})$ of $G$ with the product $\mathfrak{h}^{\sigma_{1}} \times \cdots \times \mathfrak{h}^{\sigma_{x}}$, obtained by differentiation. In this situation, we know (and can prove easily) that $p$ induces a one-to-one map of $\mathfrak{g}_{k}$ with $\mathfrak{h}_{K}$, which is an isomorphism as Lie algebras over $k$. Let $\left\{\omega_{j}\right\}_{1 \leqq j \leqq \delta}$ be a basis of $K / k$. Then $\left\{\omega_{j} X_{i}\right\}, 1 \leqq i \leqq d, 1 \leqq j \leqq \delta$, is a basis for $\mathfrak{h}_{K}$ as a vector space over $k$. Call $Z_{j i}$ the inverse image of $\omega_{j} X_{i}$ under the map $p$ restricted on $g_{k}$. Hence $\left\{Z_{j i}\right\}$ is a basis of $g_{k}$. We have

$$
\begin{aligned}
\pi\left(Z_{j i}\right) & =\left(\omega_{j} X_{i}, \omega_{j}^{\sigma_{2}} X_{i}^{\sigma_{2}}, \ldots, \omega_{j}^{\sigma_{\delta}} X_{i}^{\sigma^{\delta}}\right) \\
& =\omega_{j}\left(X_{i}, 0, \ldots 0\right)+\omega_{j}^{\sigma_{2}}\left(0, X_{i}^{\sigma_{2}}, \ldots, 0\right)+\cdots+\omega_{j}^{\sigma_{\delta}}\left(0, \cdots, X_{i}^{\sigma} \delta\right)
\end{aligned}
$$

Since $\left(0, \cdots, X_{i}^{\sigma_{\nu}}, \cdots, 0\right), 1 \leqq i \leqq d, 1 \leqq \nu \leqq \delta$, form a basis of $\mathfrak{h}^{\sigma_{1}} \times \cdots \times \mathfrak{h}^{\sigma^{\gamma}}$, denoting by $B^{\prime}, B$ the Killing forms of $\mathfrak{g}, \mathfrak{h}$, respectively, we get

$$
\operatorname{det}\left(B^{\prime}\left(Z_{j i}, Z_{j^{\prime},}\right)\right)=\left(\operatorname{det}\left(\omega_{j}^{\sigma \nu}\right)\right)^{2 d} \Pi_{\nu=1}^{\delta} \operatorname{det}\left(B\left(X_{i}, X_{j}\right)\right)^{\sigma_{\nu}} .
$$

From (2.1.19), (2.1.20), (2.1.21), we see that

$$
\mu(G / k) \equiv\left(\operatorname{det}\left(\omega_{j}^{\sigma_{\nu}}\right)\right)^{d} \Pi_{\nu=1}^{\delta} \mu(H / K)^{\sigma_{\nu}} \quad \bmod . k^{*} .
$$

If, in particular, $k=\mathbf{Q}$ and if we take as $\omega_{j}, 1 \leqq j \leqq \delta$, an integral basis of $K$, then

$$
\operatorname{det}\left(\omega_{j}^{\sigma \nu}\right)=\Delta_{K}^{1 / 2}, \quad \Delta_{K} \text { is the discriminant of } K,
$$

and hence, we have

$$
\mu(G / \mathbf{Q}) \sim \Delta_{K}^{\pi / 2} \Pi_{\nu=1}^{\delta} \mu(H / K)^{\sigma_{\nu}}, \quad \boldsymbol{d}=\operatorname{dim} H .
$$

Therefore, if $M_{H / K}=K$, we have $\mu(G / \mathbf{Q}) \sim \Delta_{K}^{d / 2}$, since $\Pi_{\nu=1}^{\delta} \mu(H / K)^{\sigma_{\nu}}=N_{K / Q} \mu(H / K)$ $\in \mathbf{Q}$. On the other hand, if $M_{H / K}$ is quadratic over $K$, by taking a basis $\left\{\omega_{i}, \rho \omega_{j}\right\}$ of $M_{H / K}$ over $\mathbf{Q}$, where $\{1, \rho\}$ is a basis of $M_{H / K}$ over $K$, we see easily that $\Pi_{\nu=1}^{\delta} \mu(H / K)^{\sigma_{\nu}} \sim \Delta_{M}^{1 / 2}, \Delta_{M}$ being the discriminant of $M=M_{H / K}$, and so we get 
Proposition 2.1.23. Let $K$ be a number field, $H$ be a connected semi-simple algebraic group defined over $K$ and let $G=R_{K / Q}(H)$. Put $M_{H / K}=K(\mu(H / K))$. Then we have

$$
\mu(G / \mathbf{Q}) \sim \begin{cases}\Delta_{K}^{(\operatorname{dim} H) / 2} & \text { if } M_{H / K}=K \\ \Delta_{K}^{(\operatorname{dim} H) / 2} \Delta_{M H / K}^{1 / 2} & \text { if }\left[M_{H / K}: K\right]=2 .\end{cases}
$$

\subsection{Discriminant $\Delta(G / \mathbf{Q})$}

Let $G$ be a connected semi-simple algebaic group defined over $\mathbf{Q}$. We simply put

$$
\Delta(G / \mathbf{Q})=|\mu(G / \mathbf{Q})|^{2} \quad\left(=N_{\mathbf{C} / \mathbf{R}} \mu(G / \mathbf{Q})\right)
$$

and call this the discriminant of $G$ over $\mathbf{Q}$, Thus, the positive rational number $\Delta(G / \mathbf{Q})$ mod. $\left(\mathbf{Q}^{*}\right)^{2}$ is invariantly attached to $G$ over $\mathbf{Q}$. From (2.1.1), (2.1.2), we get

$$
\begin{array}{ll}
\Delta\left(G \times G^{\prime} / \mathbf{Q}\right) \equiv \Delta(G / \mathbf{Q}) \Delta\left(G^{\prime} / \mathbf{Q}\right) & \bmod .\left(\mathbf{Q}^{*}\right)^{2}, \\
\Delta(G / \mathbf{Q}) \equiv \Delta\left(G^{\prime} / \mathbf{Q}\right) & \bmod .\left(\mathbf{Q}^{*}\right)^{2}
\end{array}
$$

when $G^{\prime}$ is isogenous to $G$ over $\mathbf{Q}$. Using the notation in (2.1.23), we get

$$
\Delta(G / \mathbf{Q})^{1 / 2} \sim \begin{cases}\left|\Delta_{K}\right|^{(\operatorname{dim} H) / 2} & \text { if } M_{H / K}=K \\ \left|\Delta_{K}\right|^{(\operatorname{dim} H) / 2}\left|\Delta_{M_{H / K}}\right|^{1 / 2} & \text { if }\left[M_{H / K}: K\right]:=2 .\end{cases}
$$

We shall now interpret the number $\Delta(G / \mathbf{Q})$ in connection with a compact involution of the Lie algebra $g$ of $G$. Let $\left\{X_{1}, \ldots, X_{d}\right\}$ be any basis of $g_{Q}$. Since $g$ is the complexefication of $g_{R}$, one can take a compact involution $\&$ of $g$. Let $B$ be the Killing form of $g$. One sees that the hermitian form given by

$$
B_{\iota}(X, Y)=-B(X, \imath Y), \quad X, Y \in \mathfrak{g}
$$

is positive definite. Since, leaves $g_{R}$ invariant, , induces on $g_{R}$ an automorphism $\iota_{\mathbf{R}}$ and hence $\operatorname{det} \iota_{\mathbf{R}}= \pm 1$. One has then

$$
\operatorname{det}\left(B_{i}\left(X_{i}, X_{j}\right)\right)= \pm \operatorname{det}\left(B\left(X_{i}, \iota_{\mathbf{R}} X_{j}\right)\right)= \pm \operatorname{det}\left(B\left(X_{i}, X_{j}\right)\right) .
$$

Since $\operatorname{det}\left(B_{\iota}\left(X_{i}, X_{j}\right)\right)$ is positive, we get from (2.1.18) the following

$$
\operatorname{det}\left(B_{\iota}\left(X_{i}, X_{j}\right)\right)^{1 / 2} \sim|q(g)|^{1 / 2} \Delta(G / \mathbf{Q})^{1 / 2} .
$$

Conversely, (2.2.5) will serve as an alternative definition of $\Delta(G / \mathbf{Q})$. This formula will play a role in the next section. 
Remark 2.2.6. We give here a list of the number $\Delta(G / \mathbf{Q})^{1 / 2}$ when $G=$ $R_{K / Q}(H)$ with $H$ simple. The results follow from (2.2.3) together with the determination of $M_{H / K}$ given in (2.1.10). As usual, we put $\operatorname{dim} H=l+2 N$.

Case 1. $N_{H / K}=K$

From (2.1.4) we get $M_{H / K}=K$, and so $\Delta(G / \mathbf{Q})^{1 / 2} \sim\left|\Delta_{K}\right|^{l / 2}$.

Case 2. $\left[N_{H / K}: K\right]=2$

This case can happen only for types $A_{l}(l \geqq 2), D_{l}(l \geqq 4)$ and $E_{6}$

TABLE 3

\begin{tabular}{l|c|c}
\hline \multicolumn{1}{c|}{ type } & $M_{H / K}$ & $\Delta(G / \mathbf{Q})\left(\bmod . \mathbf{Q}^{*}\right)$ \\
\hline$A_{l}(l \equiv 0,1$ mod. 4$)$ & $K$ & $\left|\Delta_{K}\right|^{l / 2}$ \\
$A_{l}(l \equiv 2,3$ mod. 4$)$ & $N_{H / K}$ & $\left|\Delta_{K}\right|^{l / 2}\left|\Delta_{N_{H / K}}\right|^{1 / 2}$ \\
$D_{l}(l \geqq 4)$ & $N_{H / K}$ & $\left|\Delta_{K}\right|^{l / 2}\left|\Delta_{N_{H / K}}\right|^{1 / 2}$ \\
$E_{6}$ & $K$ & 1
\end{tabular}

Case 3. $\left[N_{H / K}: K\right]=3$

This happens for type $D_{4}$. Again we have $M_{H / K}=K$ by (2.1.4). Hence we have $\Delta(G / \mathbf{Q})^{1 / 2} \sim 1$.

Case 4. $\left[N_{H / K}: K\right]=6$

Again $G$ is of type $D_{4}$. We have shown in (2.1.10) that $M_{H / K}$ is the unique quadratic subextension of $N_{H / K}$. Hence we get $\Delta(G / \mathbf{Q})^{1 / 2} \sim\left|\Delta_{M_{H / K}}\right|^{1 / 2}$.

\section{Homogeneous spaces}

\section{1. Compact involution}

Let $G$ be a connected semi-simple Lie group with finite center, let $\mathfrak{g}$ be the Lie algebra of $G$. For a compact involution s of $\mathfrak{g}^{\mathrm{C}}$, we have put $\mathfrak{u}_{\ell}=\left\{X \in \mathfrak{g}^{\mathrm{C}}\right.$, $\iota(X)=X\}$ and $\mathfrak{k}_{\iota}=\mathfrak{g} \cap \mathfrak{u}_{\iota}$ (cf. 1.5). We also defined the hermitian form $B_{\iota}$ on $g^{\mathrm{C}}$ by

$$
B_{\iota}(X, Y)=-B(X, \iota Y), \quad X, Y \in g^{\mathrm{C}},
$$

where $B$ is the Killing form of $g^{\mathrm{C}}$ (cf. (2.2.4)). If $B_{\iota}$ takes real values on a real subspace $\xi$ of $g^{C}$, it induces a positive definite bilinear form $\left(B_{\iota}\right)_{\S}$ on $\xi_{\text {. }}$ For a subspace $\xi^{\prime}$ of $g$ we shall denote by $g^{\prime \mathscr{E}}$ the orthogonal complement of $g^{\prime}$ with respect to $\left(B_{\ell}\right)_{3}$. For example, one can take $\xi=g$ since one has 


$$
\begin{aligned}
& B_{\iota}(X, Y)=-B(X, \iota Y)=-B\left(\iota_{0} X, \iota_{0} Y\right) \\
& =-B\left(\iota_{0} X, c_{0} Y\right)=\overline{-B(X, \bullet Y)}=\overline{B_{\iota}(X, Y)}, \quad X, Y \in \mathrm{g} .
\end{aligned}
$$

In this case, we have $\mathfrak{f}_{\iota}^{\mathfrak{g}}=\mathfrak{g} \cap \sqrt{-1} \mathfrak{u}_{\iota}$ and the direct sum $g=\mathfrak{f}_{\iota}+\mathfrak{f}_{\iota}^{\mathfrak{g}}$ is known as the Cartan decomposition of $g$ with respect to c. On the other hand, if one takes $\mathfrak{g}=\mathfrak{u}_{\ell}$, one has $\mathfrak{f}_{\ell}^{\mathfrak{t}} \iota=\sqrt{-1} \mathfrak{f}_{\ell}^{\mathfrak{g}}$ and the decomposition $\mathfrak{u}_{\ell}=\mathfrak{f}_{\ell}+\sqrt{-1} \mathfrak{f}_{\ell}^{\mathfrak{g}}$ is known as the dual of the Cartan decomposition of $g$. If two compact involutions $\iota, \iota^{\prime}$ are connected by (1.5.3), we have

$$
B_{\iota}\left(A d(g)^{\mathrm{C}}(X), A d(g)^{\mathrm{C}}(Y)\right)=B_{\iota}(X, Y), \quad X, Y \in g^{\mathrm{C}},
$$

from which follows, for example, that for a given basis $\left\{X_{1}, \ldots, X_{d}\right\}$ of $\mathrm{g}^{\mathrm{C}}$, the positive real number det $\left(B_{\imath}\left(X_{i}, X_{j}\right)\right)$ is independent of the choice of $\iota$.

\subsection{Volume element}

We first consider the following general situation. Let $G$ be a Lie group, $H$ a closed connected subgroup, $\mathfrak{g}, \mathfrak{h}$ the corresponding Lie algebras. Assume that there is a positive definite bilinear form $\beta: g \times g \rightarrow \mathbf{R}$ such that

$$
A d_{G}(H) \text { leaves } \beta \text { invariant. }
$$

Denote by $\mathfrak{h}^{\perp}$ the orthogonal complement of $\mathfrak{h}$ with respect to $\beta$. Then $\mathfrak{g}=$ $\mathfrak{h}+\mathfrak{h}^{\perp}$ (direct), and since $A_{d_{G}}(H)$ acts on $\mathfrak{h}$, it acts also on $\mathfrak{h}^{\perp}$. Let $M=G / H$ and let $\pi$ be the natural map $G \rightarrow M$. Through the identification by $d \pi$ of $\mathfrak{h}^{\perp}$ with $M_{0}$ (the tangent space at $0=\pi(e), e$ being the identity of $G$ ), the action of $A d_{G}(H)$ on $\mathfrak{h}^{\perp}$ coincides with the natural action of $H$ on $M_{0}$, and so the restriction $\beta_{\mathfrak{y}} \perp$ of $\beta$ on $\mathfrak{h}^{\perp}$ produces naturally a $G$-invariant metric $g_{M}$ on $M$. We denote by $g_{G}, g_{H}$ the metrics on $G, H$, respectively, induced by $\beta, \beta_{\mathfrak{\eta}}$ in a natural manner. We denote by $d M, d G, d H$ the corresponding volume elements. Let $\left\{X_{1}, \ldots, X_{\lambda}\right\},\left\{Y_{1}, \ldots, Y_{\mu}\right\}$ be bases of $\mathfrak{h}, \mathfrak{h}^{\perp}$ and let $\left\{Z_{1}, \ldots, Z_{\nu}\right\}$, $\nu=\lambda+\mu$, be the basis of $g$ consisting of $\left\{X_{i}, Y_{j}\right\}$, taken jointly. Let $\left\{\xi_{1}, \ldots, \xi_{\lambda}\right\}$, $\left\{\eta_{1}, \ldots, \eta_{\mu}\right\},\left\{\zeta_{1}, \ldots, \zeta_{\nu}\right\}$ be invariant 1 -forms dual to the above bases of $\mathfrak{h}, \mathfrak{h}^{\perp}, \mathfrak{g}$. Then, viewing $\eta_{i}^{\prime} s$ as forms on $M$ through the identification $\mathfrak{h}^{\perp}=M_{0}$, we get

$$
\begin{aligned}
& d H=\left(\operatorname{det}\left(\beta_{\mathfrak{\eta}}\left(X_{i}, X_{j}\right)\right)\right)^{1 / 2} \xi_{1} \wedge \cdots \wedge \xi_{\lambda} \\
& d M=\left(\operatorname{det}\left(\beta_{\mathfrak{\eta}} \perp\left(Y_{i}, Y_{j}\right)\right)\right)^{1 / 2} \eta_{1} \wedge \cdots \wedge \eta_{\mu} \\
& d G=\left(\operatorname{det}\left(\beta\left(Z_{i}, Z_{j}\right)\right)\right)^{1 / 2} \zeta_{1} \wedge \cdots \wedge \zeta_{\nu} .
\end{aligned}
$$

From these, if we use the same symbol for the positive invariant measure cor- 
responding to the volume element, we see that the three invariant measures $d G, d H, d M$ are coherent with respect to making the quotient, i.e.

$$
\int_{G} f(g) d G=\int_{M} d M \int_{H} f(g h) d H
$$

for a function $f(g)$ on $G$.

Coming back to our original situation in 3.1 , let $G$ be a connected semisimple Lie group with finite center. Fixing once for all a compact involution ` of $g^{\mathbf{c}}$, we put $\mathfrak{u}=\mathfrak{u}_{\iota}, \mathfrak{t}=\mathfrak{f}_{\iota}=\mathfrak{g} \cap \mathfrak{u}$. Denote by $K, U$ the connected compact Lie groups corresponding to $\mathfrak{k}, \mathfrak{u}$, respectively. Let $T$ be a maximal torus of $U$. As in 1.5 , we put $X=G / K, X_{u}=U / K$. We also put $V=U / T$.

Let $B$, be the positive definite hermitian form on $g^{\mathrm{C}}$ with respect to the fixed involution $\_$and let $\left(B_{\iota}\right)_{\mathfrak{g}},\left(B_{\iota}\right)_{\mathfrak{u}}$ be the positive definite bilinear forms on $\mathrm{g}, \mathfrak{u}$, respectively, induced by $B_{\iota}$. Since $\operatorname{Ad}_{G}(K)$ (resp. $A d_{U}(K)$ ) leaves $\left(B_{\iota}\right)_{\mathfrak{g}}\left(\right.$ resp. $\left.\left(B_{\iota}\right)_{\mathfrak{u}}\right)$ invariant, the procedure described above defines invariant metrics on $G, U, K, X, X_{u}, T$ and $V$. The volume elements and the corresponding invariant measures will be denoted by $d G, d U, d K, d X, d X_{u}, d T$ and $d V$. From the remark at the end of 3.1 , we see that $d G$ does not depend on the choice of a and depends only upon $B$, i.e. $d G$ is intrinsic (and so are $d U, d K$, $d X, d X_{u}, d T$ and $\left.d V\right)$. From (3.2.2) we get

$$
\int_{U} d U=\int_{X u} d X_{u} \int_{K} d K=\int_{V} d V \int_{T} d T
$$

\section{3. Generalized Euler number}

Let $M$ be an orientable riemannian manifold of even dimension $m=2 m_{0}$ with a metric $g$. Denote by $\Omega(M)$ the corresponding Euler form on $M$. We put

$$
E(M)=\int_{M} \Omega(M) .
$$

The Gauss-Bonnet theorem says that when $M$ is compact the integral is independent of the choice of $g$ and is equal to the Euler number $E(M)$ in the ordinary sense ([1], [7]). In terms of the curvature tensor $R$ on $M, \Omega(M)$ can be written as follows. Using a basis $\left\{X_{1}, \ldots, X_{m}\right\}$ for the vector fields on an open set of $M$, put

$$
R_{i j k l}=g\left(R\left(X_{i}, X_{j}\right) X_{l}, X_{k}\right), \quad 1 \leqq i, j, k, l \leqq m
$$


and

$$
\kappa(M)=\frac{1}{2^{m_{0}} m ! \operatorname{det}\left(g\left(X_{i}, X_{j}\right)\right)} \sum_{\mu, \nu} \varepsilon^{(\mu)} \varepsilon^{(\nu)} R_{\mu_{1} \mu_{2} \nu_{1} \nu_{2}} \cdots R_{\mu_{m-1} \mu_{m} \nu_{m-1} \nu_{m}},
$$

here $\sum_{\mu, \nu}$ means the summation over all indices $\mu_{i}, \nu_{j} ;$ and $\varepsilon^{(\mu)}$ is the sign function of $(\mu)=\left(\mu_{1}, \ldots, \mu_{m}\right)$. Then $\kappa(M)$ is a function on $M$ and will be called the curvature of $M$. One has then

$$
\Omega(M)=2 A_{m}^{-1} \kappa(M) d M,
$$

where $A_{m}=2^{2 m_{0}+1} m_{0} !(m !)^{-1} \pi^{m_{0}}$, the surface-area of the unit $m$-sphere, and $d M$ is the volume element of $M$ determined by $g$.

Consider now the homogeneous spaces $X, X_{u}$ and $V$ endowed with riemannian structures described in 3.2. First of all, we have $\operatorname{dim} V=2 N$, where $N$ is the number of positive roots of $g^{\mathrm{C}}$. We know that $E(V)=[W(U)]$, where $W(*)$ means the Weyl group of a compact Lie group $*([5$, II, p. 337, Th. 24.3]). Next, as for the space $X_{u}$, we always assume that

$$
E\left(X_{u}\right)>0 \text {. }
$$

This condition imples that $\operatorname{dim} X=\operatorname{dim} X_{u}=m=2 m_{0}$ and that $E\left(X_{u}\right)=[W(U)]$ / $[W(K)]([3$, p. 191 , line 5 from the bottom $\rfloor)$. Thus, all spaces $X, X_{u}$ and $V$ are of even dimension and one can define $\kappa(X), \kappa\left(X_{u}\right)$ and $\kappa(V)$. These functions are all constants because we are dealing with invariant metrics on homogeneous spaces. By virtue of the Gauss-Bonnet theorem applied to $X_{u}$, $V$ and by the relation (3.3.2) below, these constants are different from zero. Let $\pi$ be the natural map of $G$ (resp. $U$ ) onto $X$ (resp. $X_{u}$ ) and put $0=\pi(e)$. Then, $d \pi=(d \pi)_{e}$ identifies the tangent space $X_{0}$ (resp. $\left.\left(X_{u}\right)_{0}\right)$ with the subspace $\mathfrak{f}^{\mathfrak{g}}$ (resp. $\mathfrak{f}^{1 \mathrm{t}}=\sqrt{-1} \mathfrak{f}^{\mathfrak{g}}$ ) which is the orthogonal complement of $\mathfrak{f}$ with respect to $\left(B_{\iota}\right)_{\mathfrak{g}}\left(\right.$ resp. $\left.\left(B_{\iota}\right)_{\mathfrak{u}}\right)$. In this situation, the curvature tensor $R$ on $X\left(\operatorname{resp} . X_{u}\right)$ is determined by its value at 0 by the formula

$$
R_{0}(X, Y) Z=-[[X, Y], Z], \quad X, Y, Z \in \mathfrak{f}^{\mathfrak{g}}\left(\text { resp. } \mathfrak{k}^{\mathfrak{u}}\right)
$$

([11, p. 180, Th. 4.2]). From this and the definition of the curvature, we get

$$
\kappa\left(X_{u}\right)=(-1)^{m_{0}} \kappa(X) .
$$

Applying the Gauss-Bonnet theorem to compact manifolds $X_{u}$ and $V$, we get 


$$
\begin{aligned}
& \frac{2 \kappa\left(X_{u}\right)}{A_{m}} \int_{X u} d X_{u}=\frac{[W(U)]}{[W(K)]} \\
& \frac{2 \kappa(V)}{A_{2 N}} \int_{V} d V=[W(U)] .
\end{aligned}
$$

Now, let $\Gamma$ be a discrete subgroup of $G$. Then $I$ acts on $X$ properly discontinuously. We assume that

$$
\int_{\Gamma \backslash G} d G \quad \text { is finite }
$$

and that $\Gamma$ acts on $X$ without fixed points.

Because of (3.3.6), $I \backslash X$ becomes a manifold. The riemannian structure on $X$ induces naturally such a structure on $\Gamma \backslash X$; we denote again by $d X$ the volume element of $\Gamma \backslash X$. Since the structure of $X$ and $\Gamma \backslash X$ coincides locally, we have $\kappa(\Gamma \backslash X)=\kappa(X)$. In view of (3.3.5), the number $E(\Gamma \backslash X)$ is finite and is given by

$$
E(\Gamma \backslash X)=\frac{2 \kappa(X)}{A_{m}} \int_{\Gamma \backslash X} d X
$$

By a simple consideration on fundamental domains, we get from (3.2.2)

$$
\int_{\Gamma \backslash G} d G=\int_{\Gamma \backslash X} d X \int_{K} d K
$$

From $(3.3 .2),(3.3 .3),(3.3 .4),(3.3 .7),(3.3 .8)$, we get

$$
\int_{\Gamma \backslash A} d G=\frac{(-1)^{m_{0}} E(\Gamma \backslash X)[W(K)]}{[W(U)]} \int_{U} d U
$$

Furthermore, using (3.4.9) below, we get

$$
\begin{gathered}
\int_{\Gamma \backslash G} d G=\frac{(-1)^{m_{0}} 2^{l} \pi^{l+N}|q(g)|^{1 / 2}[W(K)]}{\left[\pi_{1}(U)\right][W(U)]} \Pi \underset{\alpha>0}{\frac{\langle\alpha, \alpha\rangle}{\langle\alpha, \delta\rangle} E(\Gamma \backslash X)} \\
\sim \pi^{l+N}|q(g)|^{1 / 2} E(I \backslash X),
\end{gathered}
$$

where $q(g)$ is given by $(2.1 .17)$.

\subsection{Volume of $U$}

In view of (3.2.3), we shall compute the volume of $T$ and $V$ separately. When the Lie group $G$ is compact, then $G=K=U$ and the formula (3.4.9) below will give the volume of any connected compact semi-simple Lie group 
with respect to the volume element determined canonically by the Killing form. (For this case, $\iota_{0}$ is the only compact involution and one has $\left.\left(B_{\iota_{0}}\right)_{g}=-B_{g}\right)$.

(i) Volume of $T$

Let $t$ be the Lie algebra of $T$. The map exp; $t \rightarrow T$ is a covering homomorphism with the discrete kernel $L$. The module $L$ is $Z$-free of rank $l=\operatorname{dim}$ $T$. Let $\left\{T_{1}, \ldots, T_{l}\right\}$ be a basis for $L$. By expressing $T_{i}^{\prime}$ s as linear combinations of an orthonormal basis with respect to the positive definite form $-B$ on $t$, one sees easily that

$$
\int_{T} d T=\left(\operatorname{det}\left(-B\left(T_{i}, T_{j}\right)\right)\right)^{1 / 2}
$$

As before, denote by $\langle\lambda, \mu\rangle$ the inner product on the dual space $\left({ }_{1}^{\mathbf{C}}\right)^{*}$ given by $\langle\lambda, \mu\rangle=B\left(H_{\lambda}^{\prime}, H_{\mu}^{\prime}\right)$, where $H_{\lambda}^{\prime} \in \mathrm{t}^{\mathrm{C}}$ is determined by the condition $B\left(H_{\lambda}^{\prime}, H\right)$ $=\lambda(H)$ for all $H \in \mathrm{t}^{\mathbf{C}}$. Let $\mathfrak{M}$ be the module of all weights of $\mathfrak{u}^{\mathbf{C}}$ with respect to $t^{C}$ and let $\mathfrak{M}_{U}$ be the submodule of $\mathfrak{M}$ consisting of all weights of $d \rho$ for all representations $\rho$ of $U$. Then we know that

$$
\left[\mathfrak{M}: \mathfrak{M}_{U}\right]=\left[\pi_{1}(U)\right],
$$

where $\pi_{1}(U)$ is the fundamental group of $U$. We also know that the bases $\left\{\Lambda_{1}, \ldots, \Lambda_{l}\right\},\left\{2 \pi \sqrt{-1} \lambda_{1} \ldots, 2 \pi \sqrt{-1} \lambda_{l}\right\}$ for $M, M_{U}$, respectively, are deter mined by the following orthogonality conditions

$$
\Lambda_{i}\left(\frac{2 H_{\alpha j}^{\prime}}{\left\langle\alpha_{j}, \alpha_{j}\right\rangle}\right)=\lambda_{i}\left(T_{j}\right)=\delta_{i j}, \quad 1 \leqq i, j \leqq l,
$$

here $\left\{\alpha_{i}\right\}_{1 \leqq i \leqq l}$, is a system of fundamental roots of $\mathfrak{u}^{\mathbf{C}}$ with respect to $t^{\mathrm{C}}$. The right hand side of (3.4.1) can be computed from (3.4.2), (3.4.3), and we get

$$
\int_{T} d T=\frac{(2 \pi)^{l} 2^{l / 2} C^{1 / 2}}{\left[\pi_{1}(U)\right]} \Pi_{i=1}^{l}\left\langle\alpha_{i}, \alpha_{i}\right\rangle^{-1 / 2}, \text { with } C=\operatorname{det}\left(\frac{2\left\langle\alpha_{i}, \alpha_{j}\right\rangle}{\left\langle\alpha_{j}, \alpha_{j}\right\rangle}\right) .
$$

(ii) Volume of $V=U / T$

The following computation is due to A. Orihara and I learned it through M. Ise. Let $t^{\mathfrak{u}}$ be the orthogonal complement of $t$ with respect to $\left(B_{\iota}\right)_{\mathfrak{u}}=-B_{\mathfrak{u}}$. Let $X_{\alpha}^{\prime}$ s be root vectors of $\mathfrak{u}^{\mathrm{C}}$ with respect to ${ }^{\mathrm{C}}$ which satisfy the -condition

$$
B\left(X_{\alpha}, X_{-\alpha}\right)=-1, \quad \iota\left(X_{\alpha}\right)=X_{-\alpha},
$$

and put 


$$
x_{\alpha}=\frac{1}{\sqrt{2}}\left(X_{\alpha}+X_{-\alpha}\right), \quad y_{\alpha}=\frac{\sqrt{-1}}{\sqrt{2}}\left(X_{\alpha}-X_{-\alpha}\right) .
$$

Then $\left\{x_{\alpha}, y_{\alpha}\right\}_{\alpha>0}$ forms an orthonormal basis for $1^{\mathfrak{u}}$ with respect to $-B_{\mathfrak{u}}$. Identifying $\mathfrak{t}^{\mathfrak{t}}$ with the tangent space $V_{0}$ at $0=\pi(e)$ as in 3.2 , let $\left\{\xi_{\alpha}, \eta_{\alpha}\right\}_{\alpha>0}$ be invariant 1-forms on $V$ dual to $\left\{x_{\alpha}, y_{\alpha}\right\}_{\alpha>0}$. Then $d V$ has the form

$$
d V=\Pi_{\alpha>0} \xi_{\alpha} \wedge \eta_{\alpha} .
$$

On the other hand, we know that the Euler form $\Omega(V)$ is given by

$$
\Omega(V)=\Pi_{\alpha>0} \Omega_{\alpha},
$$

where $\Omega_{\alpha}$ is the following 2 -form on $V$ :

$$
\Omega_{\alpha}=\frac{1}{2 \pi} \sum_{\beta>0}\langle\alpha, \beta\rangle \xi_{\beta} \wedge \eta_{\beta}
$$

([5, I. 10.3 and 14.5]). Hence, we get from (3.4.5)

$$
\begin{aligned}
\Omega(V) & =\frac{1}{(2 \pi)^{N}} \Pi_{\alpha>0}\left(\sum_{\beta>0}\langle\alpha, \beta\rangle \xi_{\beta} \wedge \eta_{\beta}\right) \\
& =\frac{1}{(2 \pi)^{N}}\left\{\sum_{\left(i_{1}, \ldots, i_{N}\right)}\left\langle\alpha_{1}, \alpha_{i_{1}}\right\rangle \cdots\left\langle\alpha_{N}, \alpha_{i_{N}}\right\rangle\right\} d V,
\end{aligned}
$$

where $\left(i_{1}, \ldots, i_{N}\right)$ are all permutations of $(1, \ldots, N)$. We also know that

$$
\sum_{\left(i_{1}, \ldots, i_{N}\right)}\left\langle\alpha_{1}, \alpha_{i_{i}}\right\rangle \cdots\left\langle\alpha_{N}, \alpha_{i_{N}}\right\rangle=[W(U)] \Pi_{\alpha>0}\langle\alpha, \delta\rangle,
$$

with

$$
\delta=\frac{1}{2} \sum_{\alpha>0} \alpha^{* 1}
$$

From (3.3.7), (3.4.6), (3.4.7), we get

$$
\int_{V} d V=(2 \pi)^{N} \Pi_{\alpha>0}\langle\alpha, \delta\rangle^{-1}, \quad \delta=\frac{1}{2} \sum_{\alpha>0} \alpha .
$$

Multiplying (3.4.4) by (3.4.8), we get

$$
\int_{U} d U=\frac{(2 \pi)^{l+N} 2^{l / 2} C^{1 / 2}}{\left[\pi_{1}(U)\right]}\left\langle\alpha_{i}, \alpha_{i}\right\rangle^{-1 / 2} \Pi_{\alpha>0}\langle\alpha, \delta\rangle^{-1}
$$

\section{Tamagawa number}

4.1. Definition of $\tau(G)$

Let $G$ be a connected semi-simple algebraic group defined over $\mathbf{Q}$. We

* A. Orihara informed me a proof in the framework of [6]. 
shall recall briefly the definition of the Tamagawa number $\tau(G)$ (as for the detail, see [25, Chap. II]). We shall fix an imbedding over $\mathbf{Q}$ of $G$ in a $G L(n)$ so that the definition of the group $G_{\mathbf{Z}}$ of units and the notion of the reduction modulo $p$ make sense. Let $\omega$ be a highest invariant differential form on $G$ defined over $\mathbf{Q}$. On each local group $G_{v}=G_{\mathbf{Q}^{v}}, v$ being a valuation of $\mathbf{Q}, \omega$ induces a Haar measure $\omega_{v}$. Since the product

$$
\Pi_{p} \int G_{\mathbf{Z}_{p}} \omega_{p}
$$

is absolutely convergent (see e.g. [18, Appendix II]), a Haar measure $\omega_{A}$ is well-defined on the adele group $G_{A}$, independently of the choice of $\omega$ by the product formula in $\mathbf{Q}$. It is fundamental that the volume of a fundamental domain for the discrete subgroup $G_{Q}$ in $G_{A}$ is finite ([4, Th. 5.8]). Thus a positive real number $\tau(G)$, the Tamagawa number, can be attached to $G$ by

$$
\tau(G)=\int_{\sigma_{Q} \backslash \sigma_{A}} \omega_{A}
$$

and by the construction it is easily be seen that $\tau(G)$ is independent of the choice of the imbedding of $G$ in a $G L(n)$.

\subsection{Fundamental domain}

We shall consider the fundamental domain for $G_{Q}$ in $G_{A}$ more closely. We put

$$
G_{A}^{\infty}=G_{\mathbf{R}} \times \Pi_{\nu} G_{\mathbf{Z}_{p}},
$$

this being an open subgroup of $G_{A}$. It is known that the double coset space $G_{Q} \backslash G_{A} / G_{A}^{\infty}$ consists of a finite number of elements ([4, Th, 5.1]). This number depends on the imbedding of $G$ in $G L(n)$, and will be called the class number of $G$ as a subgroup of $G L(n)$. Let $c$ be the class number and let

$$
G_{A}=\cup_{i=1}^{c} G_{\mathrm{Q}} x_{i} G_{A}^{\infty}
$$

be the disjoint decomposition of $G_{A}$ into double cosets. Since $G_{Q}$ acts independently on each coset $G_{Q} x_{i} G_{A}^{\infty}$, in order to find a fundamental domain for $G_{Q}$ in $G_{A}$, it is enough to find a fundamental domain for $G_{\mathrm{Q}}$ in $G_{\mathrm{Q}} x_{i} G_{A}^{\infty}$ for each $i$, $1 \leqq i \leqq c$. Then the union of these fundamental domains will be a fundamental domain for $G_{Q}$ in $G_{A}$.

For any adele $x \in G_{A}$, put 


$$
\Gamma_{x}=G_{\mathrm{Q}} \cap x G_{A}^{\infty} x^{-1} .
$$

It is easy to see that $\Gamma_{x}$ is discrete in $G_{\mathrm{R}}$ and commensurable with $G_{\mathrm{Z}}=\Gamma_{e}, e$ being the identity of $G_{A}$. Let $F_{x}$ be a fundamental domain for $\Gamma_{x}$ in $G_{\mathrm{R}}$ and put

$$
\widetilde{F}_{x}=F_{x} \times \Pi_{p} x_{p} G_{Z_{p}} x_{p}^{-1},
$$

where $x=\left(x_{v}\right) \in G_{A}$. It is easy to see that $\widetilde{F}_{x}$ is a fundamental domain for $G_{\mathrm{Q}}$ in $G_{\mathrm{Q}} x G_{A}^{\infty} x^{-1}$. Therefore $\widetilde{F}_{x} x$ becomes a fundamental domain for $G_{\mathrm{Q}}$ in $G_{\mathrm{Q}} x G_{A}^{\infty}$. In view of the invariance of the measure, we have

$$
\int_{\widetilde{F}_{x} x} \omega_{A}=\int_{\widetilde{F}_{x}} \omega_{A}
$$

and

$$
\int_{x_{p} \sigma \mathbf{Z}_{p} x_{p}^{-1}} \omega_{p}=\int_{G \mathbf{Z}_{p}} \omega_{p}, \quad \text { for all } p .
$$

Hence, from (4.1.1), (4.2.1), (4.2.3), we get

$$
\begin{aligned}
\tau(G) & =\sum_{i=1}^{c} \int_{\widetilde{F} x_{i} x_{i}} \omega_{A} \\
& =\sum_{i=1}^{c}\left(\int_{F_{x_{i}}} \omega_{\mathbf{R}} \Pi \int_{p} \int_{G \mathbf{Z}_{p}} \omega_{p}\right) \\
& =\left(\sum_{i=1}^{c} \int_{\Gamma_{i} \backslash \theta_{\mathbf{R}}} \omega_{\boldsymbol{R}}\right) \Pi_{p} \int_{\sigma_{\mathbf{Z}_{p}}} \omega_{p},
\end{aligned}
$$

where $\Gamma_{i}=\Gamma_{x_{i}}, 1 \leqq i \leqq c$. Since all $\Gamma_{i}$ are commensurable with $G_{\mathrm{Z}}$, we see from (4.2.3) that $G_{\mathrm{Z}} \backslash G_{\mathrm{R}}$ has a finite measure and that

$$
G_{\mathbf{Q}} \backslash G_{A} \text { is compact } \Leftrightarrow G_{\mathbf{Z}} \backslash G_{\mathrm{R}} \text { is compact. }
$$

Proposition 4.2.5. The integral $\int_{G_{Z_{p}}} \omega_{p}$ is a rational number for every $p$.

In fact, choose an imbedding $G \subset G L(\boldsymbol{n})$ and put

$$
G_{\mathbf{Z} p}^{(r)}=\left\{x \in G_{\mathbf{Z}_{p}}, x \equiv e \bmod . p^{r}\right\}
$$

where $r$ is a natural number. The group $G_{\mathbf{Z}_{p}}^{(r)}$ is normal in $G_{\mathbf{Z}_{p}}$ with finite index. Hence, to prove our assertion it is enough to show that the integral

$$
\int_{G \mathbf{Z} p}^{(r)} \omega_{p}
$$

is rational for a suitable $r$. Let $t_{1}, \ldots, t_{d}, d=\operatorname{dim} G$, be a system of local 
coordinates defined over $\mathbf{Q}$ around $e$ such that $t_{i}(e)=0,1 \leqq i \leqq d$. For sufficiently large $r$, the map $x \rightarrow\left(t_{1}(x), \ldots, t_{d}(x)\right)$ gives a homeomorphism in the sense of $p$-adic topology between $G_{\mathbf{Z} p}^{(r)}$ and a compact neighborhood $U$ of the origin in the $p$-adic space $\mathbf{Q}_{p}^{d}$. Now the given differential form $\omega$ can be expressed, around $e$, as $\omega=f(x) d t_{1} \wedge \ldots \wedge d t_{d}$, where $f(x)$ is a rational function on $G$ defined over $\mathbf{Q}$ and holomorphic at $e$. By multiplying a rational number if necessary, we may assume without loss of generality that $f(e)=1$. Then we take again $r$ large enough so that the power series expansion of $f(x)$ in the parameters $t_{i}, 1 \leqq i \leqq d$, converges in $U$ and that $|f(x)|_{p}=1$ for $x \in G_{\mathbf{Z} p}^{(r)}$. The local expression of $\omega_{p}$ on $G_{\mathbf{Z} p}^{(r)}$ is then simply $\omega_{p}=\left|d t_{1}\right|_{p} \ldots\left|d t_{d}\right|_{p}$, where $|d t|_{p}$ means the canonical measure on $\mathbf{Q}_{p}$. Hence the integral (4.2.6) is equal to the volume of $U$ by the canonical measure on $\mathbf{Q}_{p}^{d}$, which is a rational number (more precisely, a rational number whose denominator is a power of $p$ ) because the compact neighborhood $U$ is a disjoint union of a finite number of cosets in $\mathbf{Q}_{p}^{d}$ modulo various powers of $p$, q.e.d.

Remark 4.2.7. For almost all $p$, it is known that

$$
\int_{G_{\mathbf{Z}_{p}}} \omega_{p}=\frac{\left[G_{\mathrm{F}_{p}}^{\left(p_{p}\right.}\right]}{p^{i}}, \quad d=\operatorname{dim} G,
$$

where $G^{(p)}$ is the reduction modulo $p$ of $G$ ([25, Th. 2.2.5]). Hence, using the notation in 1.4 , we may write

$$
\Pi_{p}^{\prime} \int_{{ }_{\mathbf{Z}_{p}}} \omega_{p} \sim e(G / \mathbf{Q})^{-1}
$$

4.3. $\tau(G) \bmod . \mathbf{Q}^{*}$

Let $G$ be a connected semi-simple algebraic group defined over $\mathbf{Q}$ of dimension $d, \mathrm{~g}$ be the Lie algebra of $G$. As explained in $0.4, g_{\mathbf{R}}$ is identified with the Lie algebra $g_{0}$ of the Lie group $G_{0}$ which is the topological identity component of $G_{\mathrm{R}}$, and hence $\mathrm{g}=\mathrm{g}_{0}^{\mathrm{C}}$. Let $\left\{X_{1}, \ldots, X_{d}\right\}$ be a basis of $g_{\mathrm{Q}}$. Let s be a compact involution of $g$ and let $B_{\iota}$ be the positive definite hermitian form on $g$. The volume element $d G_{0}$ corresponding to the canonical riemannian metric on $G_{0}$ is given by

$$
d G_{0}=\left(\operatorname{det}\left(B_{\iota}\left(X_{i}, X_{j}\right)\right)\right)^{1 / 2} \omega_{\mathbf{R}},
$$

where $\omega_{\mathbf{R}}$ is the invariant $d$-form on $G_{0}$ which is the product of 1 -forms dual to $X_{i}, 1 \leqq i \leqq d$. Let $I$ be an arithmetic subgroup of $G$ (a subgroup of $G_{Q}$ 
commensurable with $G_{\mathrm{Z}}$ ) such that $\Gamma \subset G_{0}$ and that $\Gamma$ acts on the symmetric space $X=G_{0} / K$ without fixed points. From (4.2.4), (4.3.1) we get

$$
\tau(G) \sim\left(\int_{\Gamma \backslash \mathcal{G}_{0}} d G_{0}\right)\left(\operatorname{det}\left(B_{\iota}\left(X_{i}, X_{j}\right)\right)\right)^{-1 / 2} e(G / \mathbf{Q})^{-1} .
$$

Furthermore, from (2.2.5), (3.3.10), (4.3.2), we get

$$
\tau(G) \sim \pi^{l+N} E(\Gamma \backslash X) \Delta(G / \mathbf{Q})^{1 / 2} e(G / \mathbf{Q})^{-1}
$$

provided $E\left(X_{u}\right)>0$.

\section{Evaluation of zeta and $L$-functions}

Let $k$ be an algebraic number field and let $\mathbf{G}=S L(n), n \geqq 2$, be the special linear group viewed as an algebraic group defined over $k$. The group $\mathbf{G}$ is obviously of Chevalley type over $k$ and simple. Denote by $f$ the automorphism $x \rightarrow{ }^{t} x^{-1}$ of $\mathbf{G}$. Let $K$ be a quadratic extension of $k$ and $a, \bar{a}$ be the conjugation of $K$ over $k$. Since we have $\bar{f} f=\mathrm{id}$. ( $\in \operatorname{Aut}_{K} \mathbf{G}$ ), (id., $\left.f\right)$ is a cocycle of $g(K / k)$ in Aut $_{K} \mathbf{G}$, and hence there is a unique algebraic group $\widetilde{G}$ defined over $k$, up to isomorphisms over $k$, and an isomorphism $\phi$ of $\widetilde{G}$ with $\mathbf{G}$ defined over $K$ such that $f=\bar{\phi} \phi^{-1}$. The map $\phi$ induces an isomorphism of $(\widetilde{G})_{k}$ with the subgroup $\left\{x \in \mathbf{G}_{K},{ }^{t} x^{-1}=\bar{x}\right\}$ where the latter group is usually called the special unitary group with respect to $K / k$. We shall also call $\widetilde{G}$ the special unitary group with respect to $K / k$. Since $f$ is not an inner automorphism of $\mathbf{G}$ and $\phi$ is defined over $K$, we see at once that $K$ is the nuclear field for $\widetilde{G}$ over $k$ : $N_{\tilde{G} / k}=K$. We shall put $G=R_{k / Q}(\widetilde{G})$, this being a connected semi-simple algebraic group defined over $\mathbf{Q}$.

Now, assume that $k$ is totally real and $K$ is totally imaginary. Then, for every infinite places $\infty_{i}$ of $k,(\widetilde{G})_{k \infty_{i}}$ is isomorphic to the usual special unitary group $S U(n)$ and so it is compact. Therefore $G_{\mathrm{R}}$ is also compact and one has $E(\Gamma \backslash X)=1$, trivially. As for the numbers $l, N$ for $G$, they are $\delta(=[k: \mathbf{Q}])$ times of the corresponding numbers $\tilde{l}, \widetilde{N}$ for $\widetilde{G} ; \widetilde{l}=n-1, \widetilde{N}=n(n-1) / 2$. The square root of the discriminant $\Delta(G / \mathbf{Q})$ can be seen from Table 3 , i.e.

$$
\Delta(G / \mathbf{Q})^{1 / 2} \sim \begin{cases}\left|\Delta_{k}\right|^{(n-1) / 2} & \text { if } n \equiv 1,2 \bmod .4 \\ \left|\Delta_{k}\right|^{(n-1) / 2}\left|\Delta_{K}\right|^{1 / 2} & \text { if } n \equiv 0,3 \bmod .4 .\end{cases}
$$

Finally, the number $e(G / \mathbf{Q})(\sim e(\widetilde{G} / k)$ by (1.4.4)) is obtained from Table 2 : 


$$
e(G / \mathbf{Q}) \sim \zeta_{k}(2) L_{K / k}(3) \zeta_{k}(4) L_{K / k}(5) \cdots\left\{\begin{array}{l}
\zeta_{k}(n): n \text { even } \\
L_{K / k}(n): n \text { odd }
\end{array}\right.
$$

On the other hand, we know that

$$
\tau(G)=\tau_{k}(\widetilde{G})=1
$$

([25, Th. 4.4.1]). Substituting (5.3) in (4.3.3), we get

$$
e(G / \mathbf{Q}) \sim \pi \frac{\delta(n-1)(n+2)}{2} \Delta(G / \mathbf{Q})^{1 / 2},
$$

which holds for every $n \geqq 2$. In view of (5.1), (5.2), the system of formulas (5.4) for all $n \geqq 2$ determines the values of $\zeta_{k}(n)\left(n\right.$ : even) and $L_{K / k}(n)(n$ : odd) mod. $\mathbf{Q}^{*}$. Namely, we get the following

THEOREM 5.5. Let $K / k$ be a quadratic extension such that $k$ is totally real and $K$ is totally imaginary. Denote by $\zeta_{k}$ the zeta function of $k$ and by $L_{K / k}$ the L-function with respect to the character belonging to the quadratic extension $K / k$. Let $\Delta_{k}\left(\right.$ resp. $\left.\Delta_{K}\right)$ be the discriminant of $k(r e s p . K)$, and let $n$ be an integer $\geqq 2$. Then we have

$$
\begin{array}{ll}
\zeta_{k}(n) \sim \pi^{n[k: \mathbf{Q}]}\left|\Delta_{k}\right|^{1 / 2} & \text { if } n \text { is even } \\
L_{K / k}(n) \sim \pi^{n[k: \mathbf{Q}]}\left|\Delta_{k}\right|^{1 / 2}\left|\Delta_{K}\right|^{1 / 2} & \text { if } n \text { is odd. }
\end{array}
$$

Remark 5.8. (5.6), (5.7) were proved by Siegel [23, p. 289]. See also Klingen [12]. For a special $K / k$, Leopoldt [15] gave explicit formulas for (5.6), (5.7).

\section{Main theorem}

Let $G$ be a connected semi-simple algebraic group defined over $\mathbf{Q}$. As before, we denote by $l, N$ the rank and the number of positive roots of $G$. Assuming that $G$ is of type $(P)$ over $Q$, we want to obtain an explicit form of the number $e(G / \mathbf{Q})$. In view of (1.4.2), (1.4.3), we may assume that $G$ is Q-simple. Let $\widetilde{G}$ be any simple factor of $G$ and $k$ be the smallest field of definition for $\widetilde{G}$. Then $e(G / Q) \sim e(\widetilde{G} / k)$ by (1.4.4). Since we assumed that $G$ is of type $(P)$ over $\mathbf{Q}, \widetilde{G}$ is of type $(P)$ over $k$ by (1.5.8). Hence $k$ is totally real by (1.7.6). Let $N_{\tilde{G} / k}$ be the nuclear field for $\widetilde{G}$ over $k$. Then $N_{\tilde{G} / k}$ is totally real or totally imaginary and we have

$N_{\tilde{G} / k}$ is totally imaginary $\Longleftrightarrow \widetilde{G}$ is of type $A_{\tilde{l}}(\tilde{l} \geqq 2), D_{\tilde{l}}(\tilde{l}: \operatorname{odd} \geqq 5)$ or $E_{6}$ (cf. (1.7.6)), here and from now on $\tilde{l}, \tilde{N}$ will mean the rank and the number 
of positive roots of $\widetilde{G}$. We put $\delta=[k: \mathbf{Q}]$, and so $l=\delta \tilde{l}, N=\delta \tilde{N}$. As before, we discuss four cases separately.

Case 1: $\quad N_{\tilde{G} / k}=k$

Since $k$ is totally real, it can not be totally imaginary and hence groups of type $A_{\tilde{l}}(\tilde{l} \geqq 2), D_{\tilde{l}}(\tilde{l}:$ odd $\geqq 5)$ and $E_{6}$ must be excluded. This implies that the numbers $a_{v}, 1 \leqq \nu \leqq \tilde{l}$, appeared in the Poincare polynomial (cf (1.2.3)) are all even. Hence, by (1.4.5), (5.5), we get

$$
e(\widetilde{G} / k) \sim \Pi_{\nu=1}^{\tilde{l}} \zeta_{k}\left(a_{\nu}\right) \sim \pi^{l+N}\left|\Delta_{k}\right|^{\tilde{l} / 2}
$$

because $\tilde{l}+\tilde{N}=\sum_{\nu=1}^{\tilde{l}} a_{\nu}$.

Case 2. $\left[N_{\tilde{G} / k}: k\right]=2$

All values of $\zeta_{k}$ in Table 2 are computable $\left(\bmod . \mathbf{Q}^{*}\right)$ since $k$ is totally real and all arguments are even. As for $A_{\tilde{l}}(\tilde{l} \geqq 2), D_{\bar{l}}(\tilde{l}:$ odd $\geqq 5)$ and $E_{6}$, $N_{\tilde{G} / k}$ is totally imaginary and the $L$-functions are computable since all arguments are odd. For the case $D_{\tilde{l}}(\tilde{l}:$ even $\geqq 4) N_{\tilde{G} / k}$ is totally real and so $L_{\left(N_{\tilde{G} / k}\right) / k}(\tilde{l})$ $=\zeta_{N \tilde{G} / k}(\tilde{l}) / \zeta_{k}(\tilde{l})$ is computable. Using $(5.5)$, we get the following

TABLE 4

\begin{tabular}{|c|c|c|}
\hline type & \multicolumn{2}{|c|}{$e(G / k)\left(\bmod . \mathbf{Q}^{*}\right)$} \\
\hline$A \tau(\tilde{l} \geqq 2)$ & $\begin{array}{l}\pi^{l+N}\left|\Delta_{k}\right|^{\tilde{l} / 2}\left|\Delta_{N \widetilde{G} / k}\right|^{(\widetilde{l}-1) / 4} \\
\pi^{l+N}\left|\Delta_{k}\right|^{\tilde{l} / 2}\left|\Delta_{N} \widetilde{G / k}\right|^{\tilde{l} / 4}\end{array}$ & $\begin{array}{l}\tilde{l}: \text { odd } \\
\tilde{l}: \text { even }\end{array}$ \\
\hline$D \tilde{\imath}(\tilde{l} \geqq 4)$ & $\pi^{l+N}\left|\Delta_{k}\right|^{\tilde{l} / 2}\left|\Delta_{N} \widetilde{G} / k_{k}\right|^{1 / 2}$ & \\
\hline$E_{6}$ & $\pi^{1+N}=\pi^{42 \delta}$ & \\
\hline
\end{tabular}

Case 3. $\left[N_{\tilde{G} / k}: k\right]=3$

In this case, $N_{\tilde{G} / k}$ is totally real. From (1.4.6), we get

$$
e(\tilde{G} / k) \sim \zeta_{N \tilde{G} / k}(4) \zeta_{k}(4)^{-1} \zeta_{k}(2) \zeta_{k}(6) \sim \pi^{16 \delta}\left|\Delta_{k}\right|^{1 / 2}\left|\Delta_{N \tilde{G} / l k}\right|^{1 / 2} .
$$

Now, it is an easy exercise to show that $\left|\Delta_{N \tilde{G} / k}\right|^{1 / 2} \sim\left|\Delta_{k}\right|^{1 / 2}$. Hence, we get

$$
e(\widetilde{G} / k) \sim \pi^{16 \delta}=\pi^{l+N} \text {. }
$$

Case 4. $\left[N_{\tilde{G} / k}: k\right]=6$

The field $N_{\tilde{G} / k}$ is again totally real, and so is the quadratic subfield $M_{\tilde{G} / k}$. From (1.4.7), we get 


$$
\begin{aligned}
\boldsymbol{e}(\widetilde{G} / k) & \sim \zeta_{N \tilde{G} / k}(4)^{1 / 2} \zeta_{M \tilde{G} / k}(4)^{-1 / 2} \zeta_{k}(2) \zeta_{k}(6) \\
& \sim \pi^{16 \delta} \sqrt{r}=\pi^{l+N} \sqrt{r}, \quad r \in \mathbf{Q},
\end{aligned}
$$

here we used again the fact that $\left|\Delta_{N_{\tilde{G} / k}}\right|^{1 / 2} \sim\left|\Delta \Delta_{\tilde{G} / k}\right|^{1 / 2}$.

Combining (6.1), Table 4, (6.2), (6.3) with the corresponding values of $\Delta(G / \mathbf{Q})^{1 / 2}$ in (2.2.6), we get, for $\mathbf{Q}$-simple group $G$,

$$
\pi^{l+N} \Delta(G / \mathbf{Q})^{1 / 2} e(G / \mathbf{Q})^{-1} \sim 1
$$

except the case where $\left[N_{\tilde{\sigma} / k} ; k\right]=6$. For this exceptional case, we get

$$
\pi^{l+N} \Delta(G / \mathbf{Q})^{1 / 2} e(G / \mathbf{Q})^{-1} \sim \sqrt{q}, \quad q \in \mathbf{Q} .
$$

Let $\widetilde{G}$ be a simple group defined over $\overline{\mathbf{Q}}$ of type $D_{4}$ and $k$ be the smallest field of definition for $\widetilde{G}$. By abuse of language, we shall $\widetilde{G}$ a trialitarian form of the first (resp. second) kind over $k$ if $\left[N_{\tilde{G} / k}: k\right]=3$ (resp. $=6$ ). From (4.3.3), (6.5), we get the following

TheOREM 6.6. Let $G$ be a connected semi-simple algebraic group defined over $\mathbf{Q}, G_{0}$ be the topological identity component of $G_{\mathbf{R}}$ and $X$ be the symmetric space attached to $G_{0}$. Let $\Gamma$ be an arithmetic subgroup of $G$ such that $\Gamma \subset G_{0}$ and that $\Gamma$ acts on $X$ without fixed points. If $G$ is of type $(P)$ over $\mathbf{Q}$ and if $G$ does not contain a simple factor which is a trialitarian form of the second kind of type $D_{4}$ over its smallest field of definition, then we have

$$
\tau(G) \sim E(\Gamma \backslash X) .
$$

If, in particular, $G_{\mathbf{Z}} \backslash G_{\mathrm{R}}$ is compact, then $\tau(G)$ is a rational number.

Remark 6.7. Notation being as in (6.6), if $X$ is hermitian symmetric, then $G$ is of type $(P)$ over $\mathbf{Q}$ : this can be seen from [11, Prop. 5.5, Th. 6.1, pp. 310-311]. Furthermore, by looking at Cartan's list of irreducible symmetric spaces ([11, p. 3̄4, Table 2]) we see that every $\mathbf{Q}$-simple group of type $E_{\overline{7}}, E_{8}$, $F_{4}, G_{2}$ is of type $(P)$ over $\mathbf{Q}$ if the smallest field of definition for a simple factor is totally real.

Remark 6.8. It is quite likely that $E(\Gamma \backslash X)$ is rational even for the case where $G_{\mathbf{Z}} \backslash G_{\mathbf{R}}$ is not compact as Satake has shown for symplectic groups ([21]). We hope to come back to this question some time in the future.

Remark 6.9. When $G$ is a $\mathbf{Q}$ simple group whose simple factor is a trialitarian form of second kind of type $D_{4}$ over its smallest field of definition, 
then (6.6) must be modified as

$$
\tau(G) \sim \sqrt{q} E(\Gamma \backslash X), \quad q \in \mathbf{Q} .
$$

Let $\widetilde{G}$ be a simple factor of $G, k$ be the smallest field of definition for $\widetilde{G}$. Put $N=N_{\tilde{G} / k}, M=M_{\tilde{G} / k}$, the latter being the quadratic subextension of $N$. If $G$ is of type $(P)$ over $\mathbf{Q}$, all fields $N, M, k$ are totally real. Define rational numbers $\rho_{M}, \rho_{N}$ by

$$
\begin{aligned}
& \zeta_{N}(4)=\rho_{N} \pi^{4[N: \mathbf{Q}]}\left|\Delta_{N}\right|^{1 / 2} \\
& \zeta_{M}(4)=\rho_{M} \pi^{4[M: \mathbf{Q}]}\left|\Delta_{M}\right|^{1 / 2},
\end{aligned}
$$

and put $\rho_{N / M}=\left|\Delta_{N}\right|^{1 / 2}\left|\Delta_{M}\right|^{-1 / 2}$, this being a rational number. Then,

$$
e(G / \mathbf{Q}) \sim e(\widetilde{G} / k) \sim\left(\rho_{N} \rho_{M}^{-1} \rho_{N / M}\right)^{1 / 2} \pi^{16[k: \mathbf{Q}]} .
$$

From (6.5), we see that

$$
\sqrt{q} \sim 1 \Longleftrightarrow \rho_{N} \rho_{M} \rho_{N / M} \equiv\left|\Delta_{M}\right| \quad \bmod .\left(\mathbf{Q}^{*}\right)^{2} .
$$

In order to prove the right hand side of (6.9), more detailed study of the numbers $\rho_{N}, \rho_{M}$ will be required. In terms of $L$-function, we can restate (6.9) as

$$
\sqrt{\boldsymbol{q}} \sim 1 \Longleftrightarrow L_{N / M}(\chi, 4) \sim \pi^{4[M: \mathbf{Q}]}\left|\Delta_{M}\right|^{1 / 3},
$$

where $\chi$ is a cubic character belonging to $N / M$.

Remark 6.12. If we do not stick to the Tamagawa number, we can restate (6.6) as follows. Namely, let $\Gamma$ be any discrete subgroup of $G_{\mathbf{R}}$ such that $\Gamma \backslash G_{\mathbf{R}}$ is of volume finite. Put $\Gamma^{\prime}=\Gamma \cap G_{\mathbf{R}}$. The space $\Gamma^{\prime} \backslash X$ is not a manifold, in general, but a $V$-manifold. However, the Euler form still makes sense and one can define $E(I \backslash X)$ in the same manner as in 3.3. Accordingly, (3.3.10) still holds for this case*!. Then the proof of (6.6) shows that

$$
\int_{\Gamma \backslash G_{\mathbf{R}}} \omega_{\mathbf{R}} \sim E(\Gamma \backslash X) e(G / \mathbf{Q}) .
$$

If, in particular, $\Gamma \backslash G_{\mathbf{R}}$ is compact, so is $\Gamma^{\prime} \backslash X$ and $E\left(I^{\prime} \backslash X\right)$ is rational, the Euler number in the sense of Satake [21]. Hence, we get

$$
\int_{\Gamma \backslash G_{\mathbf{R}}} \omega_{\mathbf{R}} \sim e(G / \mathbf{Q}),
$$

* The only thing we have to modify is to divide the right hand side of (3.3.10) by [ $\left.\Gamma_{0}\right]$ where $\Gamma_{0}$ is the finite normal subgroup of $\Gamma^{\prime}$ consisting of all $\gamma \in \Gamma^{\prime}$ which act trivially on $X$. 
this is the formula $(*)$ in the introduction because $e(G / \mathbf{Q})$ can be expressed as a product of $L$-functions as shown in (1.4) and one can replace $\omega$ by $a \omega$ with any $\boldsymbol{a} \in \mathbf{Q}^{*}$.

\section{REFERENCES}

[1] C. B. Allendoerfer and A. Weil, The Gauss-Bonnet theorem for Riemannian polyhedra, Trans. A mer. Math. Soc. 53 (1943), $10-129$.

[2] P. Alexadroff and H. Hopf, Topologie I, Berlin, 1935.

[3] A. Borel, Sur la cohomologie des espaces fibrés principaux et des espaces homogènes de groupes de Lie compacts. Ann. of Math. 57 (1953), 115-207.

[ 4 ] A. Borel, Some finiteness properties of adele groups over number fields, I.H.E.S. No. 16, 1963, 101-126.

[5] A. Borel and F. Hirzebruch, Characteristic classes and homogeneous spaces, I, Amer. J. Math. 80 (1958), 459-538, II, ibid. 81 (1959), 315-382.

[6] Harish-Chandra, Differential operators on a semi-simple Lie algebra. Amer. J. Math. 79 (1957), 87-120.

[7] S. S. Chern, A simple intrinsic proof of the Gauss-Bonnet formula for closed Riemannian manifolds, Ann. of Math. 45 (1944), 747-752.

[ 8 ] C. Chevalley, Théorie des groupes de Lie II, Paris, 1951.

[9] C. Chevalley, Sur certains groupes simples, Tôhoku Math. J. 7 (1955), 14-66.

[10] Séminaire C. Chevalley, Classification des groupes de Lie algébriques, E.N.S., 1958.

[11] S. Helgason, Differential geometry and symmetric spaces, Academic Press, New York, 1962.

[12] H. Klingen, Über die Werte der Dedekindschen Zetafunktion, Math. Annalen 145 (1962), 265-272.

[13] S. Lang, Algebraic groups over finite fields, Amer. J. Math. 78 (1956), 555-563.

[14] Séminaire S. Lie, E.N.S., 1955.

[15] H. W. Leopoldt, Eine Verallgemeinerung der Bernoullischen Zahlen, Abh. Math, Sem. Hamburg, 22 (1958), 131-140.

[16] T. Ono, Sur les groupes de Chevalley, J. Math. Soc. Japan 10 (1958), 307-313.

[17] T. Ono, On the field of definition of Borel subgroups of semi-simple algebraic groups, J. Math. Soc. Japan 15 (1963), 392-395.

[18] T. Ono, On the relative theory of Tamagawa numbers, Ann. of Math. 82 (1965),88-111.

[19] R. Ree, On some simple groups defined by C. Chevalley, Trans. Amer. Math. Soc. 84 (1957), 392-400.

[20] H. Samelson, Topology of Lie groups, Bull. Amer. Math. Soc. 58 (1952), 2-37.

[21] I. Satake, The Gauss-Bonnet theorem for V-manifolds, J. Math. Soc. Japan 9 (1957), 464-492.

[22] C. L. Siegel, Symplectic geometry, Amer. J. Math. 65 (1943), 1-86.

[23] C. L. Siegel, Über die analytische Theorie der quadratischen Formen III. Ann. of Math. 38 (1937), 212-291.

[24] R. Steinberg, Variations on a theme of Chevalley, Pacific J. of Math. 9 (1959), 875891.

[25] A. Weil, Adeles and algebraic groups, The Institute for Advanced Study, Princeton N.J., 1961.

\section{University of Pennsylvania}

